\title{
On the structure theory of the Iwasawa algebra of a $p$-adic Lie group
}

Received May 12, 2001 / final version received July 5, 2001

Published online September 3, 2001 - (C) Springer-Verlag \& EMS 2001

\begin{abstract}
This paper is motivated by the question whether there is a nice structure theory of finitely generated modules over the Iwasawa algebra, i.e. the completed group algebra, $\Lambda$ of a $p$-adic analytic group $G$. For $G$ without any $p$-torsion element we prove that $\Lambda$ is an Auslander regular ring. This result enables us to give a good definition of the notion of a pseudo-null $\Lambda$-module. This is classical when $G=\mathbb{Z}_{p}^{k}$ for some integer $k \geq 1$, but was previously unknown in the non-commutative case. Then the category of $\Lambda$-modules up to pseudo-isomorphisms is studied and we obtain a weak structure theorem for the $\mathbb{Z}_{p}$-torsion part of a finitely generated $\Lambda$-module. We also prove a local duality theorem and a version of Auslander-Buchsbaum equality. The arithmetic applications to the Iwasawa theory of abelian varieties are published elsewhere.
\end{abstract}

\section{Introduction}

The increasing interest in the Iwasawa algebra, i.e. the completed group algebra

$$
\Lambda(G)=\mathbb{Z}_{p} \llbracket G \rrbracket
$$

of a compact $p$-adic analytic group $G$ stems at least from the following two reasons: Firstly, in a purely algebraic sense $\Lambda(G)$ is a prototype of a "regular" - in general non-commutative - ring. It seems very likely that the category of finitely generated $\Lambda(G)$-modules, which we denote by $\Lambda(G)$-mod, has a very rich structure, and our aim is to give some initial evidence in this direction. Secondly, compact $p$-adic Lie groups occur naturally in arithmetic geometry as the Galois groups of certain infinite extensions $k_{\infty}$ of a number field $k$, for example, $k_{\infty}$ could be taken to be the field obtained by adjoining to $k$ the coordinates of all $p$-power division points on some abelian variety defined over $k$. It is then natural to study certain arithmetic objects defined over $k_{\infty}$ (for example, the $p$-Hilbert class field of $k_{\infty}$ or the Pontryagin dual of the Selmer group of an abelian variety over $k_{\infty}$ ) as modules over the Iwasawa algebra $\Lambda(G)$ (cf. [16], [10], [9]). Though this last aspect widely motivated our study

O. Venjakob: Universität Heidelberg, Mathematisches Institut, Im Neuenheimer Feld 288, 69120 Heidelberg, Germany, e-mail: otmar@mathi.uni-heidelberg.de,

http://www . mathi . uni-heidelberg.de/ otmar/

Mathematics Subject Classification (2000): 16D70, 16E30, 16E65, 16S34 
of $\Lambda(G)$ the results of this paper are "purely algebraic". For a detailed discussion of its arithmetic applications we refer the reader to [29] and a forthcoming paper [35].

Let us assume for simplicity that the $p$-adic analytic group $G$ is torsion-free and a pro- $p$-group. Then, $\Lambda(G)$ is a (both left and right) Noetherian ring without zero-divisors. Furthermore, by results of Brumer it is known that $\Lambda(G)$ has finite projective dimension equal to $\operatorname{pd}(\Lambda(G))=\operatorname{dim}(G)+1$, where $\operatorname{dim}(G)$ denotes the dimension of $G$ as $p$-adic analytic manifold and agrees with its $p$-cohomological dimension. Thus in some sense $\Lambda(G)$ can be considered as a "regular" ring, and it is natural to ask if there could exist a structure theory for finitely generated $\Lambda(G)$ modules, which is parallel to the classical theory (studied in detail in Bourbaki) when $G=\mathbb{Z}_{p}^{k}$ for some integer $k \geq 1$. A first basic step in this direction is to answer the following question

What is a good definition of pseudo-null resp. pseudo-isomorphism in the context of $\Lambda(G)$-modules?

We recall that for a commutative Noetherian ring $R$ and a finitely generated $R$ module $M$ the definition is the following: The dimension of $M$ is defined to be the Krull dimension of the support of $M$ in $\operatorname{Spec}(R)$ and $M$ is said to be pseudo-null, if its codimension is greater than 1 . M. Harris $[16,1.12]$ already proposed a vague definition of pseudo-null using a certain filtration of $\Lambda(G)$, which in general differs from the $\mathfrak{M}$-adic one, where $\mathfrak{M}$ denotes the maximal ideal of $\Lambda(G)$, and cannot be described easily. Besides some more or less trivial cases it turned out very difficult to verify whether a module is pseudo-null.

In this article we give an answer to this question using the following strategy. First, we establish a dimension theory for finitely generated $\Lambda(G)$-modules. Our approach here has been inspired by the work of Björk [4], who showed that each finitely generated module over an Auslander regular or more generally Auslander Gorenstein ring (for the definitions see 3.3) is intrinsically equipped with a canonical filtration

$$
\mathrm{T}_{0}(M) \subseteq \mathrm{T}_{1}(M) \subseteq \cdots \subseteq \mathrm{T}_{d-1}(M) \subseteq \mathrm{T}_{d}(M)=M
$$

Using this filtration he defines the dimension of a $\Lambda$-module $M$. It turns out that for a commutative regular local ring this dimension equals the Krull dimension and that $\mathrm{T}_{i}(M)$ is just the maximal submodule of $M$ with dimension less or equal to $i$.

Thus the following theorem states a fundamental structure property of $\Lambda(G)$ and will be crucial for the applications in Iwasawa theory ([35]). Here and in what follows we assume that $G$ is a compact $p$-adic analytic group with no element of order $p$ (but not necessarily pro- $p$ ).

Theorem (Theorem 3.26). $\Lambda(G)$ is an Auslander regular ring.

For the purpose of studying the $\mathbb{Z}_{p}$-torsion part of $\Lambda(G)$-module the following consequence for the completed group algebra $\mathbb{Z} / p^{n} \llbracket G \rrbracket \cong \Lambda / p^{n}$ with coefficients in $\mathbb{Z} / p^{n}$ becomes very useful. 


\section{Theorem (Theorem 3.30).}

(i) $\mathbb{Z}_{p} / p^{m} \llbracket G \rrbracket$ is an Auslander-Gorenstein ring with injective dimension equal to $\operatorname{cd}_{p}(G)$.

(ii) $\mathbb{F}_{p} \llbracket G \rrbracket$ is an Auslander regular ring of dimension $\operatorname{cd}_{p}(G)$.

If one is ready to neglect the $\mathbb{Z}_{p}$-torsion part - which can be controlled by the last theorem and a further result below, anyway - then it might be convenient to consider also modules over the ring $\mathbb{Q}_{p} \llbracket G \rrbracket:=\mathbb{Z}_{p} \llbracket G \rrbracket \otimes_{\mathbb{Z}_{p}} \mathbb{Q}_{p}$.

Theorem (Theorem 3.29). $\mathbb{Q}_{p} \llbracket G \rrbracket$ is an Auslander regular ring of dimension $\operatorname{cd}_{p}(G)$.

Using these results, it is quite obvious how to define pseudo-null:

A finitely generated $\Lambda$-module is called pseudo-null if and only if its codimension is greater or equal to 2 .

In the case $G=\mathbb{Z}_{p}^{k}$ this is just the usual definition. So we are convinced that our definition is the right generalization to the non-commutative case.

Once having available the notation of pseudo-null modules it is natural to ask whether there holds a structure theorem of $\Lambda(G)$-modules up to pseudo-isomorphism which is analogous to the well known structure theorem for finitely generated modules over a commutative regular ring.

The first result in this direction was obtained by the author in his thesis [34], and determines the $\Lambda(G)$-structure of the $\mathbb{Z}_{p}$-torsion submodule of a finitely generated $\Lambda(G)$-module in the quotient category of $\Lambda$-mod by the Serre subcategory of pseudo-null $\Lambda$-modules. We write $\mathcal{P N}$ for the full subcategory of $\Lambda$-mod consisting of the pseudo-null modules.

Theorem (Theorem 3.40). Assume that $G$ is a p-adic analytic group without $p$ torsion such that both $\Lambda=\Lambda(G)$ and $\Lambda / p$ are integral. Then, for any $\Lambda$-module $M$, there exist uniquely (up to order) determined natural numbers $n_{1}, \ldots, n_{r}$ such that

$$
\operatorname{tor}_{\mathbb{Z}_{p}} M \equiv \bigoplus_{1 \leq i \leq r} \Lambda / p^{n_{i}} \bmod \mathcal{P N} .
$$

Both the hypotheses that $\Lambda(G)$ and $\Lambda(G) / p \Lambda(G)$ have no zero divisors are known to be true if $G$ is a uniform, pro- $p p$-adic Lie group with no element of order $p$ ([12]). Our results have inspired various mathematicians to generalize this result and search for a full analogue of the structure theory. Firstly, Susan Howson has generalized it to the submodule of $M$ annihilated by some power of a fixed prime element in the centre of $\Lambda(G)$, obtaining the stronger result that a pseudo-isomorphism exists in the category $\Lambda$-mod ([20]). Secondly, J. Coates and R. Sujatha ([11]) and P. Schneider [31] have proven that, in the quotient category $\Lambda$-mod $/ \mathcal{P N}$, every finitely generated torsion $\Lambda(G)$-module is isomorphic to a direct sum of cyclic $\Lambda(G)$-modules, when $G$ is any extra-powerful pro- $p$ p-adic Lie group with no element of order $p$. Schneider has shown that this theorem is in fact a consequence of a general result of Chamarie [8] on the structure of modules over non-commutative Krull domains, whereas Coates and Sujatha show that it can also 
be proven using techniques from the algebraic theory of micro-localization. It is still unknown whether the left cyclic modules which occur can be chosen to be quotients of $\Lambda(G)$ by left principal ideals.

Now we want to state two further main results on the structure of $\Lambda(G)$, if $G$ is a pro-p Poincaré group of finite cohomological dimension and such that $\Lambda=\Lambda(G)$ is Noetherian. The first result tells us that $\Lambda(G)$ "admits local duality à la Grothendieck", i.e. if local cohomology is defined in an natural way (see Sect. 5), we obtain

Theorem (Theorem 5.6). For any $M \in \Lambda(G)$-mod, there are canonical isomorphisms

$$
\mathrm{E}^{i}(M) \cong \operatorname{Hom}_{\Lambda}\left(\mathrm{H}_{\mathfrak{M}}^{d-i}(M), \mathrm{H}_{\mathfrak{M}}^{d}(\Lambda)\right) \cong \mathrm{H}_{\mathfrak{M}}^{d-i}(M)^{\vee}
$$

where $d=\operatorname{cd}_{p}(G)+1$.

The second result generalizes the Auslander-Buchsbaum equality.

Theorem (Theorem 6.2). For any $M \epsilon \Lambda$-mod, it holds

$$
\operatorname{pd}_{\Lambda}(M)+\operatorname{depth}_{\Lambda}(M)=\operatorname{depth}_{\Lambda}(\Lambda) .
$$

\section{Basic properties of $\Lambda$-modules}

\subsection{Preliminaries}

The aim of this work is to give some complements to the theory of $\Lambda$-modules, where we denote by $\Lambda=\Lambda(G)$ the completed group algebra of a profinite group $G$ over $\mathbb{Z}_{p}$

$$
\Lambda(G)=\mathbb{Z}_{p} \llbracket G \rrbracket=\underbrace{\lim }_{U} \mathbb{Z}_{p}[G / U]
$$

Here $U$ runs through the open normal subgroups of $G$. We start by recalling some well-known facts concerning $\Lambda$, proofs of which can be found in [27, V§2]. By a (left) $\Lambda$-module $M$ we understand a separated topological module, i.e $M$ is a Hausdorff topological $\mathbb{Z}_{p}$-module with a continuous $G$-action. Since the involution of $\Lambda$ given by passing to the inverses of group elements induces a natural equivalence between the categories of left and right $\Lambda$-modules, we will often ignore the difference without further mention. The category $\mathcal{C}=\mathcal{C}(G)$ of compact $\Lambda$-modules and the category $\mathcal{D}=\mathcal{D}(G)$ of discrete $\Lambda$-modules will be of particular interest. Both are abelian categories, and Pontryagin duality defines a contravariant equivalence of categories between them. Hence, while $\mathcal{C}$ has sufficiently many projectives and exact inverse limits the dual statement holds for $\mathcal{D}$.

By $I_{G}$ we denote the augmentation ideal of $\Lambda$, i.e. the kernel of the canonical epimorphism

$$
\mathbb{Z}_{p} \llbracket G \rrbracket \rightarrow \mathbb{Z}_{p}
$$

and by

$$
M_{G}=M / I_{G} M
$$


the module of coinvariants of $M$. Then, the $G$-homology $\mathrm{H}_{\bullet}(G, M)$ of a compact $\Lambda$-module $M$ can be defined as left derived functor of $-{ }_{G}$ or alternatively as $\operatorname{Tor}_{\bullet}^{\Lambda}\left(\mathbb{Z}_{p}, M\right)$, where Tor denotes the left derived functor of the complete tensor product $-\widehat{\otimes}_{\Lambda}-$. We obtain a canonical isomorphism $\mathrm{H}_{i}(G, M) \cong \mathrm{H}^{i}\left(G, M^{\vee}\right)^{\vee}$, where $\mathrm{H}^{\bullet}(G,-)$ denotes the usual $G$-cohomology for a discrete $\Lambda$-module considered as a discrete abelian group and ${ }^{\vee}$ is the Pontryagin dual.

In order to state the topological Nakayama lemma we define the $\operatorname{radical} \operatorname{Rad}_{G}$ of $\Lambda$ to be the intersection of all open left maximal ideals. It is a closed two-sided ideal and its powers define a topology on $\Lambda$ which is called the $R$-topology. If a $p$-Sylow group $G_{p}$ is of finite index in $G$, then this topology coincides with the canonical one [27, 5.2.16], $\operatorname{Rad}_{G}$ is an open ideal of $\Lambda$ and all (left) maximal ideals are open. Furthermore, $\Lambda(G)$ is a local ring if and only if $G$ is a pro- $p$-group. In this case the maximal ideal of $\Lambda$ is equal to $p \Lambda+I_{G}$.

\section{Lemma 1.1 (Topological Nakayama Lemma).}

(i) If $M \in \mathcal{C}$ and $\operatorname{Rad}_{G} M=M$, then $M=0$.

(ii) Assume that $G$ is a pro-p-group, i.e. $\Lambda$ a local ring with maximal ideal $\mathfrak{M}$. Then the following holds:

(a) $M$ is generated by $x_{1}, \ldots, x_{r}$ if and only if $x_{i}+\mathfrak{M} M, i=1, \ldots, r$, generate $M / \mathfrak{M} M$ as $\mathbb{F}_{p}$-vector space.

(b) Let $P \in \mathcal{C}$ be finitely generated. Then $P$ is $\Lambda$-free if and only if $P$ is $\Lambda$-projective.

Compare [2] for a thorough discussion.

Concerning the projective dimension $\operatorname{pd}_{\Lambda} M$, respectively global dimension $\operatorname{pd}(\Lambda)$ of $\Lambda$, which are both defined with respect to the category $\mathcal{C}$, there are the following results due to Brumer [6], where $\operatorname{cd}_{p}(G)$ denotes the $p$-cohomological dimension of $G$ :

$$
\operatorname{pd}_{\Lambda} \mathbb{Z}_{p}=\operatorname{cd}_{p}(G) \text { and } \operatorname{pd}(\Lambda)=\operatorname{cd}_{p}(G)+1 .
$$

If $\Lambda$ is Noetherian (e.g. if $G$ is a $p$-adic Lie group, see below), the forgetful functor from the category $\mathcal{C}$ of compact $\Lambda$-modules to the category $\Lambda$-Mod of abstract $\Lambda$-modules defines an equivalence between the full subcategory $\mathcal{C}_{f g}$ of finitely generated compact $\Lambda$-modules and the full subcategory $\Lambda$-mod of finitely generated abstract $\Lambda$-modules. In particular, the different definitions of the projective, respectively global dimension coincide in this case.

\section{2. p-adic Lie groups}

In this subsection we recall some facts about (compact) $p$-adic Lie groups or also called (compact) $p$-adic analytic groups, i.e. the group objects in the category of $p$-adic analytic manifolds over $\mathbb{Q}_{p}$. There is a famous characterization of them due to Lazard [25] (see also [12] 9.36):

A topological group $G$ is a compact p-adic Lie group if and only if $G$ contains a normal open uniformly powerful pro-p-subgroup of finite index. 
Let us briefly recall the definitions: A pro- $p$-group $G$ is called powerful, if $[G, G] \subseteq$ $G^{p}$ for odd $p$, respectively $[G, G] \subseteq G^{4}$ for $p=2$ holds. A (topologically) finitely generated powerful pro- $p$-group $G$ is uniform if the $p$-power map induces isomorphisms

$$
P_{i}(G) / P_{i+1}(G) \stackrel{. p}{\rightarrow} P_{i+1}(G) / P_{i+2}(G), \quad i \geq 1,
$$

where $P_{i}(G)$ denotes the lower central $p$-series given by

$$
P_{1}(G)=G, P_{i+1}(G)=P_{i}(G)^{p}\left[P_{i}(G), G\right], i \geq 1,
$$

(for finitely generated pro- $p$-groups). It can be shown that for a uniform group $G$ the sets $G^{p^{i}}:=\left\{g^{p^{i}} \mid g \in G\right\}$ form subgroups and in fact $G^{p^{i}}=P_{i+1}(G), i \geq 0$. For example, all the congruence kernels of $G L_{n}\left(\mathbb{Z}_{p}\right), S L_{n}\left(\mathbb{Z}_{p}\right)$ or $P G L_{n}\left(\mathbb{Z}_{p}\right)$ are uniform pro- $p$-groups for $p \neq 2$, in particular the lower central $p$-series of the first congruence kernel corresponds precisely to the higher congruence kernels. We should mention also the following basic result (see [12], p. 62):

A pro-p powerful group is uniform if and only if it has no element of order $p$.

It is a remarkable fact that the analytic structure of a $p$-adic Lie group is already determined by its topological group structure. Also, the category of $p$-adic analytic groups is stable under the formation of closed subgroups, quotients and group extensions (See [12], Chap. 10, for these facts). The following cohomological property is indispensable (for the definition of Poincaré groups see [27]).

A p-adic Lie group of dimension d (as p-adic analytic manifold) without p-torsion is a Poincaré group at $p$ of dimension $d$.

With respect to the completed group algebra we know that $\Lambda(G)$ is Noetherian for any compact $p$-adic Lie group (see [25] V 2.2.4). If, in addition, $G$ is uniform, then $\Lambda(G)$ is an integral domain, i.e. the only zero-divisor in $\Lambda(G)$ is 0 ([25]). In fact the latter property also holds for any $p$-adic analytic group without elements of finite order (see [28]). For instance, for $p \geq n+2$, the group $G l_{n}\left(\mathbb{Z}_{p}\right)$ has no elements of order $p$, in particular, $G L_{2}\left(\mathbb{Z}_{p}\right)$ contains no elements of finite $p$-power order if $p \geq 5$ (see [18] 4.7).

In this case (i.e. $\Lambda$ is both left and right Noetherian and without zero-divisors) we can form a skew field $Q(G)$ of fractions of $\Lambda$ (see [15]). This allows us to define the rank of a $\Lambda$-module:

Definition 1.2. The $\operatorname{rank}_{\mathrm{rk}_{\Lambda}} M$ is defined to be the dimension of $M \otimes_{\Lambda} Q(G)$ as a left vector space over $Q(G)$

$$
\mathrm{rk}_{\Lambda} M=\operatorname{dim}_{Q(G)}\left(M \otimes_{\Lambda} Q(G)\right) .
$$

\section{Homotopy theory of modules}

In this section we briefly recall some definitions and results from the homotopy theory of modules in the setting of U. Jannsen [22], who developed further the homotopy theory which was introduced by Eckmann and Hilton and extended by 
Auslander and Bridger [1]. The proofs can be found in [22, §1] or in [27, V§4]. Though this theory works in much larger generality, we restrict ourselves to the case where $\Lambda$ is a Noetherian (= right and left Noetherian) ring (= associative unital ring). Furthermore, all $\Lambda$-modules considered are assumed to be finitely generated.

Definition 2.1. A morphism $f: M \rightarrow N$ of $\Lambda$-modules is homotopic to zero, if it factors through a projective module $P$ :

$$
f: M \rightarrow P \rightarrow N
$$

Two morphisms $f, g$ are homotopic ( $f \simeq g$ ), if $f-g$ is homotopic to zero. Let $[M, N]=\operatorname{Hom}_{\Lambda}(M, N) /\{f \simeq 0\}$ be the group of homotopy classes of morphisms from $M$ to $N$, and let $\operatorname{Ho}(\Lambda)$ be the category, whose objects are the objects of $\Lambda$-mod and whose morphism sets are given by $\operatorname{Hom}_{H o(\Lambda)}(M, N)=[M, N]$, i.e. the category of " $\Lambda$-modules up to homotopy."

Remark 2.2. The additive homotopy category of modules is not abelian in general. It can be shown that it forms a closed model category (for suitable definitions of (co)fibrations and weak equivalences). In general, it cannot be extended to a triangulated category: If it were a triangulated category in general there would have to exist for any module $M$ a weak equivalence between $M$ and $\Omega M$, where $\Omega$ denotes the loop space functor which will be introduced below. But for a ring $\Lambda$ with finite projective dimension this would imply that all modules in $\Lambda$-mod are projective.

However, if $\Lambda$ is a quasi-Frobenius ring (for the definition and properties see $[36,4.2])$, e.g. the group algebra of a finite group over a field $\Lambda=k[G]$, then its associated homotopy category is triangulated ([14, IV Examples 4-8]).

It turns out that $M$ and $N$ are homotopy equivalent, $M \simeq N$, i.e isomorphic in $\mathrm{Ho}(\Lambda)$, if and only if $M \oplus P \cong N \oplus Q$ with projective $\Lambda$-modules $P$ and $Q$. In particular, $M \simeq 0$ if and only if $M$ is projective.

Definition 2.3. For $M \epsilon \Lambda$-mod we define the higher adjoints of $M$ to be

$$
\mathrm{E}^{i}(M):=\operatorname{Ext}_{\Lambda}^{i}(M, \Lambda), \quad i \geq 0,
$$

which are a priori right $\Lambda$-modules by functoriality and the right $\Lambda$-structure of the bi-module $\Lambda$. By convention we set $\mathrm{E}^{i}(M)=0$ for $i<0$. The $\Lambda$-dual $\mathrm{E}^{0}(M)$ will also be denoted by $M^{+}$. If $\Lambda=\Lambda(G)$ is a completed group algebra these adjoints will be considered as left modules via the involution of $\Lambda$.

Remark 2.4. In case $\Lambda$ is the completed group algebra of a profinite group $G$ we call the $E^{i}(M)$ also Iwasawa adjoints of $M$ as $E^{1}(M)$ is isomorphic (up to the inversion of the group action) to Iwasawa's adjoint $\alpha(M)$ for $G \cong \mathbb{Z}_{p}$.

It can be shown that for $i \geq 1$ the functor $\mathrm{E}^{i}$ factors through $\operatorname{Ho}(\Lambda)$ defining a functor

$$
\mathrm{E}^{i}: \operatorname{Ho}(\Lambda) \rightarrow \bmod -\Lambda
$$


Now we will describe the construction of a contravariant duality functor, the transpose

$$
\mathrm{D}: \operatorname{Ho}(\Lambda) \rightarrow \operatorname{Ho}(\Lambda)
$$

where we denote the homotopy category of right $\Lambda$-modules also by $\operatorname{Ho}(\Lambda)$. For every $M \in \Lambda$-mod choose a presentation $P_{1} \rightarrow P_{0} \rightarrow M \rightarrow 0$ of $M$ by projectives and define the transpose $\mathrm{D} M$ by the exactness of the sequence

$$
0 \longrightarrow M^{+} \longrightarrow P_{0}^{+} \longrightarrow P_{1}^{+} \longrightarrow \mathrm{D} M \longrightarrow 0 .
$$

Then it can be shown that the functor $\mathrm{D}$ is well-defined and one has $\mathrm{D}^{2}=\mathrm{Id}$. Furthermore, if $\operatorname{pd}_{\Lambda} M \leq 1$ then $\mathrm{D} M \simeq \mathrm{E}^{1}(M)$.

Sometimes it is also convenient to have the notation of the $1^{\text {st }}$ syzygy or loop space functor $\Omega: \Lambda$-mod $\rightarrow \operatorname{Ho}(\Lambda)$ which is defined as follows (see $[22,1.5]$ ): Choose a surjection $P \rightarrow M$ with $P$ projective. Then $\Omega M$ is defined by the exact sequence

$$
0 \longrightarrow \Omega M \longrightarrow P \longrightarrow M \longrightarrow 0 .
$$

The next result will be of particular importance:

Proposition 2.5. For $M \epsilon \Lambda$-mod there is a canonical exact sequence

$$
0 \longrightarrow \mathrm{E}^{1} \mathrm{D} M \longrightarrow M \stackrel{\phi_{M}}{\longrightarrow} M^{++} \longrightarrow \mathrm{E}^{2} \mathrm{D} M \longrightarrow 0,
$$

where $\phi_{M}$ is the canonical map from $M$ to its bi-dual. In the following we will refer to the sequence as "the" canonical sequence (of homotopy theory).

A $\Lambda$-module $M$ is called reflexive if $\phi_{M}$ is an isomorphism from $M$ to its bi-dual $M \cong M^{++}$.

As Auslander and Bridger suggest the module $\mathrm{E}^{1} \mathrm{D} M$ should be considered as torsion submodule of $M$. Indeed, if $\Lambda$ is a Noetherian integral domain this submodule coincides exactly with the set of torsion elements tor $_{\Lambda} M$.

Definition 2.6. A $\Lambda$-module $M$ is called $\Lambda$-torsion module if $\phi_{M} \equiv 0$, i.e. if tor $_{\Lambda} M:=\mathrm{E}^{1} \mathrm{D} M=M$. We say that $M$ is $\Lambda$-torsion-free if $\mathrm{E}^{1} \mathrm{D} M=0$.

Since $M^{++}$embeds into a free $\Lambda$-module (just take the dual of an arbitrary surjection $\Lambda^{m} \rightarrow M^{+}$) the torsion-free $\Lambda$-modules are exactly the submodules of free modules. A different characterization of torsion(-free) modules will be given later using dimension theory, see 3.7.

For $\Lambda:=\Lambda(G)$, where $G$ is a $p$-adic Lie group, the above definition can be interpreted as follows: A finitely generated $\Lambda$-module $M$ is a $\Lambda$-torsion module if and only if $M$ is a $\Lambda\left(G^{\prime}\right)$-torsion module (in the strict sense) for some open pro- $p$ subgroup $G^{\prime} \subseteq G$ such that $\Lambda\left(G^{\prime}\right)$ is integral. Indeed, for any open subgroup $H$ of a $p$-adic Lie group $G$ there is an isomorphism $\mathrm{E}_{\Lambda(G)}^{1} \mathrm{D}_{\Lambda(G)} \cong \mathrm{E}_{\Lambda(H)}^{1} \mathrm{D}_{\Lambda(H)}$ of $\Lambda(H)$-modules by Proposition 2.7 (ii) (see below) and the analogue statement for $\mathrm{D} M$.

For a closed subgroup $H \subseteq G$ we denote by $\operatorname{Ind}_{G}^{H}(M)=M \widehat{\otimes}_{\Lambda(H)} \Lambda(G)$ the compact induction of a $\Lambda(H)$-module to a $\Lambda(G)$-module. 
Proposition 2.7. Let $H$ be a closed subgroup of $G$.

(i) For any $M \in \Lambda(H)$-mod and any $i$ we have an isomorphism of $\Lambda$-modules

$$
\mathrm{E}_{\Lambda(G)}^{i}\left(\operatorname{Ind}_{G}^{H} M\right) \cong \operatorname{Ind}_{G}^{H} \mathrm{E}_{\Lambda(H)}^{i}(M) .
$$

(ii) If, in addition, $H$ is an open subgroup, then there is an isomorphism of $\Lambda(H)$ modules

$$
\mathrm{E}_{\Lambda(G)}^{i}(M) \cong \mathrm{E}_{\Lambda(H)}^{i}(M)
$$

Proof. The first statement is proved in [29, Lemma 5.5] while the second one can be found in [22, Lemma 2.3].

\section{Auslander regularity}

\subsection{Filtrations of $\Lambda$-modules}

Since the completed group ring $\Lambda$ of a $p$-adic Lie group without $p$-torsion is (both left and right) Noetherian and has finite global homological (and therefore finite injective) dimension we can apply the results of J.-E. Björk [4], which we will describe in this section.

Let $\Lambda$ be a (not necessarily commutative) Noetherian ring with finite injective dimension $d$, i.e $d$ is the minimal integer with respect to the property that $\mathrm{E}^{j}(M)=0$ for all (left and right) $\Lambda$-modules $M$ and integers $j>d$. Of course, this is equivalent to the condition that both the left and the right $\Lambda$-module $\Lambda$ has (bounded) injective dimension $d$. It can be shown that these left and right injective dimensions are the same (see [38]). The analogous statement that the left and the right global homological dimension are the same is a consequence of the Tor-dimension theorem [36, 4.1.3].

In this section all $\Lambda$-modules are assumed to be finitely generated.

Since projective $\Lambda$-modules are reflexive, we get the equality

$$
M=\mathbf{R} \operatorname{Hom}(\mathbf{R H o m}(M, \Lambda), \Lambda)
$$

for left (or right) $\Lambda$-modules $M$ in the derived category of complexes of $\Lambda$ modules (more generally, this equality holds for all perfect complexes). Calculating $\mathbf{R H o m}(\mathbf{R H o m}(M, \Lambda), \Lambda)$ by the bidualizing complex, the associated filtrations give rise to two convergent spectral sequences (see [26] for the convergence), the first of which degenerates. The second one becomes

$$
E_{2}^{p, q}=\mathrm{E}^{p}\left(\mathrm{E}^{-q}(M)\right) \Rightarrow \mathrm{H}^{p+q}\left(\Delta^{\bullet}(M)\right),
$$

where $\Delta^{\bullet}(M)$ is a filtered complex, which is exact in all degrees except zero: $\mathrm{H}^{0}\left(\Delta^{\bullet}\right)=M$, i.e. there is a canonical filtration

$$
\mathrm{T}_{0}(M) \subseteq \mathrm{T}_{1}(M) \subseteq \cdots \subseteq \mathrm{T}_{d-1}(M) \subseteq \mathrm{T}_{d}(M)=M
$$


on every module $M$ (The natural numbering of this filtration arising from the double complex is descending but we found it more convenient to reverse it). The convergence of the spectral sequence implies

$$
E_{\infty}^{p, q}= \begin{cases}\mathrm{T}_{d-p}(M) / \mathrm{T}_{d-p-1}(M) & \text { if } p+q=0, \\ 0 & \text { otherwise. }\end{cases}
$$

(By convention, $\mathrm{T}_{i}(M)=0$ for $\left.i<0\right)$.

Definition 3.1. (i) The number $\delta:=\min \left\{i \mid \mathrm{T}_{i}(M)=M\right\}$ is called the dimension $\delta(M)$ of a $\Lambda$-module $M \neq\{0\}$. We set $\delta(\{0\})=-\infty$.

(ii) If $M$ is a $\Lambda$-module we say that it has pure $\delta$-dimension if $\mathrm{T}_{\delta-1}(M)=0$, i.e. the filtration degenerates to a single term $M$.

(iii) A $\Lambda$-module $M$ is called pseudo-null, if it is at least of codimension 2, i.e. if $\delta(M) \leq d-2$.

By Grothendieck's local duality theorem, this definition coincides with the Krull dimension of $\operatorname{supp}_{\Lambda}(M)$ if $\Lambda$ is a commutative local Noetherian Gorenstein ring, see [7, Corollary 3.5.11].

First we want to state some basic facts on the $\delta$-dimension. The functoriality of the spectral sequence implies

Proposition 3.2. (i) If $0 \longrightarrow M^{\prime} \longrightarrow M \longrightarrow M^{\prime \prime} \longrightarrow 0$ is an exact sequence of $\Lambda$-modules then

$$
\mathrm{T}_{i}\left(M^{\prime}\right) \subseteq \mathrm{T}_{i}(M) \text { for all } i
$$

and $\delta\left(M^{\prime \prime}\right) \leq \delta(M)$.

(ii) $\mathrm{T}_{i}\left(\bigoplus_{k} M_{k}\right)=\bigoplus_{k} \mathrm{~T}_{i}\left(M_{k}\right)$ and $\delta\left(\bigoplus_{k} M_{k}\right)=\max _{k} \delta\left(M_{k}\right)$.

In order to analyze this spectral sequence more closely, the Auslander condition (for not necessarily commutative rings) is essential:

Definition 3.3. (i) If $M \neq 0$ is a $\Lambda$-module, then

$$
j(M):=\min \left\{i \mid \mathrm{E}^{i}(M) \neq 0\right\}
$$

is called the grade of $M$. By convention, $j(\{0\})=\infty$.

(ii) A Noetherian ring $\Lambda$ is called Auslander-Gorenstein ring if it has finite injective dimension and the following Auslander condition holds: For any $\Lambda$ module $M$, any integer $m$ and any submodule $N$ of $\mathrm{E}^{m}(M)$, the grade of $N$ satisfies $j(N) \geq m$.

(iii) A Noetherian ring $\Lambda$ is called Auslander regular ring if it has finite global homological dimension and the Auslander condition holds.

Remark 3.4. Let $\Lambda$ be a commutative ring. Then $\Lambda$ is Auslander-Gorenstein if and only if it is Gorenstein (in the usual sense). Similarly, $\Lambda$ is Auslander regular if and only if it is regular (in the usual sense) and of finite Krull dimension. (The implications concerning the injective, respectively global homological dimensions are well known, for the Auslander condition see [1, Corollary 4.6, Proposition 4.21]). 
In the next section we will prove that $\Lambda=\Lambda(G)$ is Auslander regular for any $p$-adic Lie group without $p$-torsion. Generally, for this kind of rings we get the following properties:

Proposition 3.5. Let $\Lambda$ be an Auslander regular ring and $M$ a $\Lambda$-module. Then

(i) (a) For all $i$, there is an exact sequence of $\Lambda$-modules

$$
0 \longrightarrow \mathrm{T}_{i}(M) / \mathrm{T}_{i-1}(M) \longrightarrow \mathrm{E}^{d-i} \mathrm{E}^{d-i}(M) \longrightarrow Q_{i}(M) \longrightarrow 0,
$$

where $Q_{i}(M)$ is a subquotient of $\bigoplus_{k \geq 1} \mathrm{E}^{d-i+k+1} \mathrm{E}^{d-i+k}(M)$.

(b) $\mathrm{T}_{0}(M)=\mathrm{E}^{d} \mathrm{E}^{d}(M)$ and $\mathrm{T}_{1}(M) / \mathrm{T}_{0}(M)=\mathrm{E}^{d-1} \mathrm{E}^{d-1}(M)$.

(c) $\mathrm{T}_{i}(M) / \mathrm{T}_{i-1}(M)=0$ if and only if $\mathrm{E}^{d-i} \mathrm{E}^{d-i}(M)=0$.

(ii) $\delta(M)+j(M)=d$ for $M \neq 0$.

(iii) (a) $j\left(\mathrm{E}^{i}(M)\right) \geq i$, i.e. $\mathrm{E}^{j} \mathrm{E}^{i}(M)=0$ for all $j<i$.

(b) $\delta\left(\mathrm{E}^{i}(M)\right) \leq d-i$.

(c) $\mathrm{E}^{j(M)}(M)$ has pure $\delta$-dimension $\delta(M)$.

(iv) $\mathrm{E}^{k+j(M)+1} \mathrm{E}^{k+j(M)} \mathrm{E}^{j(M)}(M)=0$ for all $k \geq 1$.

(v) (a) For all $0 \leq i \leq d, \mathrm{E}^{i} \mathrm{E}^{i}(M)$ is either zero or of pure $\delta$-dimension $d-i$.

(b) $M$ has pure $\delta$-dimension if and only if $\mathrm{E}^{i} \mathrm{E}^{i}(M)=0$ for all $i>j(M)$.

(vi) (a) $\delta\left(\mathrm{T}_{i}(M)\right) \leq i$.

(b) $\mathrm{T}_{i}(M)$ is the maximal submodule of $M$ with $\delta$-dimension less or equal to $i$.

(c) The functor $\mathrm{T}_{i}$ is left exact.

(d) $\mathrm{T}_{i}\left(M / \mathrm{T}_{i}(M)\right)=0$.

(vii) If $\delta(M)=0$ then $M$ has finite length.

Proof. Except for (i)(a), (i)(b) and (vi), these properties are all proved in [4] or trivial: Proposition 1.21, 1.16, Proposition 1.18, Remark before 1.19, Corollary 1.20, Corollary 1.22 and 1.27, while (i)(a) is proved in [26, Corollary 4.3].

The assertion (i)(b) is clear, as $E_{\infty}^{i,-i}=E_{2}^{i,-i}$ because of (iii)(a). So let us prove (vi): By (iii), (a) is equivalent to $j\left(\mathrm{~T}_{i}(M)\right) \geq d-i$ and this is true because of the Auslander condition using induction (cf. the proof of (iii)). The assertion (b) follows by Proposition 3.2: If $N$ is a submodule of dimension $\delta(N) \leq i$, then $N=\mathrm{T}_{i}(N) \subseteq \mathrm{T}_{i}(M)$.

Noting Proposition 3.2 (i), we only have to show $N \cap \mathrm{T}_{i}(M) \subseteq \mathrm{T}_{i}(N)$ in order to prove left exactness. Since the first module has dimension $\delta\left(\bar{N} \cap \mathrm{T}_{i}(M)\right) \leq i$, this is a consequence of (b).

By (c) the exact sequence

$$
0 \rightarrow \mathrm{T}_{i+1}(M) / \mathrm{T}_{i}(M) \rightarrow \mathrm{T}_{i+2}(M) / \mathrm{T}_{i}(M) \rightarrow \mathrm{T}_{i+2}(M) / \mathrm{T}_{i+1}(M) \rightarrow 0
$$

induces the exact sequence

$$
0 \rightarrow \mathrm{T}_{i}\left(\mathrm{~T}_{i+1}(M) / \mathrm{T}_{i}(M)\right) \rightarrow \mathrm{T}_{i}\left(\mathrm{~T}_{i+2}(M) / \mathrm{T}_{i}(M)\right) \rightarrow \mathrm{T}_{i}\left(\mathrm{~T}_{i+2}(M) / \mathrm{T}_{i+1}(M)\right) .
$$

The first and third term are zero by (i) and (iii) as above. Hence (d) follows by induction. 
Assuming the Auslander-condition, Proposition 3.2 can be sharpened as follows [5, Proposition 1.8]:

Proposition 3.6. Let $0 \longrightarrow M^{\prime} \longrightarrow M \longrightarrow M^{\prime \prime} \longrightarrow 0$ be an exact sequence of $\Lambda$-modules.

(i) If $\Lambda$ is Auslander-Gorenstein, then

$$
j(M)=\min \left\{j\left(M^{\prime}\right), j\left(M^{\prime \prime}\right)\right\}
$$

holds.

(ii) If $\Lambda$ is Auslander regular, then

$$
\delta(M)=\max \left\{\delta\left(M^{\prime}\right), \delta\left(M^{\prime \prime}\right)\right\}
$$

holds.

For the second assertion we used Proposition 3.5 (ii).

Remark 3.7. (i) Using the methods of [13], Proposition 6, one can show the existence of the following exact sequences:

$$
0 \rightarrow \mathrm{E}^{i+1} \mathrm{D} \Omega^{i} \mathrm{~T}_{d-i}(M) \rightarrow \mathrm{T}_{d-i}(M) \rightarrow \mathrm{E}^{i} \mathrm{E}^{i}(M) \rightarrow \mathrm{E}^{i+2} \mathrm{D} \Omega^{i} \mathrm{~T}_{d-i}(M) \rightarrow 0 .
$$

Hence, $\mathrm{T}_{i}(M)$ can also be obtained recursively by the formula $\mathrm{T}_{d-i-1}(M)=$ $\mathrm{E}^{i+1} \mathrm{D} \Omega^{i} \mathrm{~T}_{d-i}(M)$ and similarly, we get a description for $Q_{d-i}(M) \cong$ $\mathrm{E}^{i+2} \mathrm{D} \Omega^{i} \mathrm{~T}_{d-i}(M)$. The same arguments yield for a $\Lambda$-module $M$ with $j(M) \geq j$ the isomorphisms

$$
\mathrm{E}^{j+k} \mathrm{E}^{j}(M) \cong \mathrm{E}^{j+k+2} \mathrm{D} \Omega^{j}(M) \text { for } k \geq 1 .
$$

(ii) In particular, $\mathrm{T}_{d-1}(M)=\mathrm{E}^{1} \mathrm{D}(M)=\operatorname{tor}_{\Lambda} M$, i.e. the torsion submodule of $M$ is the maximal submodule of codimension greater or equal than 1 . That means that $M$ is $\Lambda$-torsion if and only if it is at least of codimension 1 , and $\Lambda$-torsion-free if and only if $M$ is of pure dimension $d$.

The following class of $\Lambda$-modules satisfies some duality relations:

Definition 3.8. A $\Lambda$-module $M \neq 0$ is called Cohen-Macaulay if $j(M)=\operatorname{pd}_{\Lambda}(M)$ holds, i.e. if $\mathrm{E}^{i}(M)=0$ for all $i \neq j(M)$.

Proposition 3.9. Let $\Lambda$ be an Auslander regular ring.

(i) Let $M$ be a Cohen-Macaulay module of dimension $j$. Then

$$
\mathrm{E}^{j} \mathrm{E}^{j}(M)=M
$$

(ii) In particular, if $\delta(M)=0$, then

$$
\mathrm{E}^{d} \mathrm{E}^{d}(M)=M .
$$

Proof. In both cases the spectral sequence degenerates. 
One could hope that any $\Lambda$-module $M$ can be decomposed into Cohen-Macaulay modules in the following sense: there is an filtration of $M$ such that the $i$ th subquotient is Cohen-Macaulay of dimension $i$. But it is easy to construct counterexamples which show that in general such a filtration does not exist. Nevertheless, there is a different type of filtration: Auslander and Bridger proved the existence of a spherical filtration (up to homotopy, i.e. after adding a projective summand $P$ )

$$
M_{d} \subseteq M_{d-1} \subseteq \cdots \subseteq M_{1} \subseteq M_{0}=M \oplus P,
$$

the subquotients of which form spherical or Eilenberg-MacLane modules of type $\mathrm{E}^{i}(M)$, i.e. for $1 \leq i \leq d$

$$
\mathrm{E}^{j}\left(M_{i-1} / M_{i}\right) \cong\left\{\begin{array}{ll}
\mathrm{E}^{i}(M) & \text { if } j=i \\
0 & \text { if } j \neq i, 0
\end{array} .\right.
$$

Fossum [13] compared the spherical filtration to the filtration $\mathrm{T}_{i}(M)$ for a commutative Gorenstein ring and proved ([13], Proposition 9) that their "torsion parts" agree for $i<d$

$$
\begin{aligned}
\mathrm{T}_{i}(M) & \cong \mathrm{T}_{d-1}\left(M_{d-i-1}\right) \\
& \cong \mathrm{T}_{i}\left(M_{k}\right) \text { for all } k<d-i .
\end{aligned}
$$

The proof generalizes at once to the non-commutative case.

Proposition 3.10. Let $\Lambda$ be an Auslander regular ring. A $\Lambda$-module $M$ with projective dimension $\operatorname{pd}_{\Lambda}(M)=k$ has no non-trivial submodule of dimension less or equal to $d-k-1$, i.e. $\mathrm{T}_{d-k-1}(M)=0$.

Proof. See Proposition 3.5, (i)(b).

The next result extends a well known result for commutative regular rings (see for example [27], Corollary 5.1.3).

Proposition 3.11. Let $\Lambda$ be an Auslander regular ring.

(i) For any $\Lambda$-module $M$, the module $\mathrm{E}^{0}(M)$ is reflexive:

$$
\mathrm{E}^{0}(M) \cong \mathrm{E}^{0} \mathrm{E}^{0} \mathrm{E}^{0}(M) .
$$

(ii) Assume that $d \geq 2$ and $\delta(M)=d-i$. Then

$$
\mathrm{E}^{i}(M) \cong \mathrm{E}^{i} \mathrm{E}^{i} \mathrm{E}^{i}(M)
$$

Proof. Let $N:=\mathrm{E}^{0}(M)$ and apply Proposition 3.5 (iv) to conclude that $\bigoplus_{k \geq 1} \mathrm{E}^{k+1} \mathrm{E}^{k}(N)=\bigoplus_{k \geq 1} \mathrm{E}^{k+1} \mathrm{E}^{k} \mathrm{E}^{0}(M)=0$, i.e. $Q_{d}(N)=0$. Since we already know by (iii)(c) that $N$ is of pure dimension $d$ (if $N \neq 0$ ), the statement (i) follows considering (i)(a). The proof of (ii) is analogous.

Corollary 3.12. For any $i$ it holds

(i) $\mathrm{E}^{i} \mathrm{E}^{i} \mathrm{E}^{i} \mathrm{E}^{i}(M) \cong \mathrm{E}^{i} \mathrm{E}^{i}(M)$ and

(ii) $\mathrm{E}^{i} \mathrm{E}^{i} \mathrm{~T}_{d-i}(M) \cong \mathrm{E}^{i} \mathrm{E}^{i}(M)$. 
Proof. To prove (i) assume first that $\delta\left(\mathrm{E}^{i}(M)=d-i\right.$. Applying the proposition to the module $\mathrm{E}^{i}(M)$ gives the result while in the second case, i.e. $j\left(\mathrm{E}^{i}(M)>i\right.$, the module $\mathrm{E}^{i} \mathrm{E}^{i}(M)$ is zero anyway. Noting that $j\left(Q_{i}(M)\right) \geq i+2$, the second assertion follows at once calculating the long exact $\mathrm{E}^{i}$-sequence of

$$
0 \longrightarrow \mathrm{T}_{d-i-1}(M) / \mathrm{T}_{d-i}(M) \longrightarrow \mathrm{E}^{i} \mathrm{E}^{i}(M) \longrightarrow Q_{i} \longrightarrow 0 .
$$

\subsection{Modules up to pseudo-isomorphisms}

As in the commutative case we say that a homomorphism $\varphi: M \rightarrow N$ of $\Lambda$-modules is a pseudo-isomorphism if its kernel and cokernel are pseudo-null. A module $M$ is by definition pseudo-isomorphic to a module $N$, denoted

$$
M \sim N,
$$

if and only if there exists a pseudo-isomorphism from $M$ to $N$. In general, is not symmetric even in the $\mathbb{Z}_{p}$-case (cf. [27, V§3, Example 1]). While in the commutative case $\sim$ is symmetric at least for torsion modules (see the first remark after Proposition 5.17 in [27]), we do not know whether this property still holds in the general case.

If we want to reverse pseudo-isomorphisms, we have to consider the quotient category $\Lambda$-mod $/ \mathcal{P N}$ with respect to subcategory $\mathcal{P} \mathcal{N}$ of pseudo-null $\Lambda$-modules, which is a Serre subcategory, i.e. closed under subobjects, quotients and extensions. By definition, this quotient category is the localization $(\mathcal{P} \mathcal{I})^{-1} \Lambda-\bmod$ of $\Lambda$-mod with respect to the multiplicative system $\mathcal{P} \mathcal{I}$ consisting of all pseudo-isomorphisms (see [36, Example 10.3.2]). Since $\Lambda$-mod is well-powered, i.e. the family of submodules of any module $M \epsilon \Lambda$-mod forms a set, these localization exists, is an abelian category and the universal functor $q: \Lambda-\bmod \rightarrow \Lambda-\bmod / \mathcal{P N}$ is exact (see [33, p. 44ff]). Furthermore, $q(M)=0$ in $\Lambda-\bmod / \mathcal{P N}$ if and only if $M \in \mathcal{P N}$.

Note that for any pseudo-isomorphism $f: M \rightarrow N$ the induced homomorphism $\mathrm{E}^{1}(f)$ is an pseudo-isomorphism (with respect to an analogue subcategory, also denoted by $\mathcal{P N}$, of $\bmod -\Lambda$ ), too. By the universal property of the localization, we obtain a functor

$$
E^{1}(-): \Lambda-\bmod / \mathcal{P N} \rightarrow \bmod -\Lambda / \mathcal{P N},
$$

which is exact if it is restricted to the full subcategory $\Lambda-\bmod ^{\geq 1} / \mathcal{P N}$ of $\Lambda-\bmod / \mathcal{P} \mathcal{N}$ consisting of all $\Lambda$-modules of codimension greater or equal to 1 . The next result shows that

$$
\mathrm{E}^{1} \circ \mathrm{E}^{1} \cong i d: \Lambda-\bmod ^{\geq 1} / \mathcal{P N} \rightarrow \Lambda-\bmod ^{\geq 1} / \mathcal{P N}
$$

is a natural isomorphism of functors:

Proposition 3.13. Let $\Lambda$ be an Auslander regular ring.

(i) Any torsion-free module $M$ embeds into a reflexive module with pseudo-null cokernel.

(ii) Any torsion module $M$ is pseudo-isomorphic to $\mathrm{E}^{1} \mathrm{E}^{1}(M)$. 
Proof. Observe that $\mathrm{T}_{d-1}(M)$ is the maximal torsion submodule in this case. Hence, the exact sequence in (i) (a) for $i=d$ respectively $i=d-1$ proves both statements taking under consideration (iii)(b) and Proposition 3.11.

Corollary 3.14. A homomorphism $f: M \rightarrow N$ of $\Lambda$-torsion modules is a pseudoisomorphism if and only if the induced homomorphism $\mathrm{E}^{1}(f): \mathrm{E}^{1}(N) \rightarrow \mathrm{E}^{1}(M)$ is.

Since $\Lambda$ is Noetherian it follows readily that any object in $\Lambda-\bmod ^{\geq 1} / \mathcal{P N}$ has the ascending chain condition (A.C.C.) (see [33, II. Chap. 2]). But using the natural isomorphism $\mathrm{E}^{1} \circ \mathrm{E}^{1} \cong i d$ its immediate that also the descending chain condition (D.C.C.) holds in this category. A consequence of this observation is that any object in $\Lambda-\bmod ^{\geq 1} / \mathcal{P N}$ has finite length. Moreover, the Krull-Schmidt-Theorem holds (loc. cit. Theorem 2.2):

Proposition 3.15. Let $\Lambda$ be an Auslander regular ring. Then any object $q(M) \epsilon$ $\Lambda-\bmod ^{\geq 1} / \mathcal{P N}$ decomposes uniquely (up to isomorphism) into a finite direct sum of indecomposable objects $q\left(M_{i}\right)$ :

$$
M \equiv \bigoplus_{i} M_{i} \bmod \mathcal{P} \mathcal{N}
$$

Proposition 3.16. Let $\Lambda$ be an Auslander regular ring. Then the following holds.

(i) The simple objects of $\Lambda-\bmod ^{\geq 1} / \mathcal{P N}$ are cyclic, i.e. of the form $q(\Lambda / I)$ for some left ideal I of $\Lambda$.

(ii) There is an isomorphism of abelian groups

$$
K_{0}\left(\Lambda-\bmod ^{\geq 1} / \mathcal{P N}\right) \cong \bigoplus_{S \in \mathcal{I}} \mathbb{Z}[S]
$$

where $\mathcal{I}$ denotes the set of isomorphism classes of simple objects of $\Lambda-\bmod ^{\geq 1} / \mathcal{P N}$.

The class $[M]$ of a 1 -codimensional $\Lambda$-module $M$ in $K_{0}\left(\Lambda-\bmod ^{\geq 1} / \mathcal{P} \mathcal{N}\right)$ should be thought of as "generalized characteristic ideal." At least if $\Lambda$ is a commutative regular ring, this class bears the same information as the characteristic ideal which is associated with $M$ via the structure theory of $\Lambda$-modules up to pseudo-isomorphism (see $[27,5.1 .7,5.18])$.

Proof. Let $M$ be a non-pseudo-null $\Lambda$-module such that $q(M)$ is a simple object. Then there exists a $m \in M$ such that $\Lambda m \cong \Lambda / a n n_{\Lambda}(m)$ is not pseudo-null, either. Consequently, $0 \neq q(\Lambda m) \subseteq q(M)$ and by the simplicity of $q(M)$ this inclusion cannot be strict. Taking $I=a n n_{\Lambda}(m)$, this proves (i) while (ii) is just [33, II Chap. 1, Theorem 1.7].

Following the structure theory for modules over a commutative regular local ring (see $[27,5.1 .7,5.18]$ ), it is natural to hope that the following question has an affirmative answer 
Question 3.17. Let $\Lambda$ be an Auslander regular ring and $M \epsilon \Lambda$-mod. Does there exist an isomorphism in $\Lambda-\bmod / \mathcal{P N}$

$$
M \cong \operatorname{tor}_{\Lambda} M \oplus R \bmod \mathcal{P N},
$$

where $R \cong M /$ tor $_{\Lambda} M \bmod \mathcal{P} \mathcal{N}$ is a reflexive $\Lambda$-module?

At least for the Iwasawa algebra $\Lambda(G)$ of an extra-powerful (for the definition see the next subsection) and uniform pro- $p$-group this should follow from the techniques used by Coates-Sujatha ([11]) to prove the structure theorem for torsion modules.

Proposition 3.18. Let $\Lambda$ be an Auslander regular ring. For any $\Lambda$-module $M$ it holds:

$$
\mathrm{E}^{1}(M) \sim \mathrm{E}^{1}\left(\operatorname{tor}_{\Lambda} M\right)
$$

Proof. From the long exact Ext-sequence we get the exact sequence

$$
\mathrm{E}^{1}\left(M / \operatorname{tor}_{\Lambda} M\right) \longrightarrow \mathrm{E}^{1}(M) \longrightarrow \mathrm{E}^{1}\left(\operatorname{tor}_{\Lambda} M\right) \longrightarrow \mathrm{E}^{2}\left(X / \operatorname{tor}_{\Lambda} M\right) .
$$

While the module on the right hand side is obviously pseudo-null the first one is so by the following argument: the long exact Ext-sequence of

$$
0 \longrightarrow M / \text { tor }_{\Lambda} M \longrightarrow \mathrm{E}^{0} \mathrm{E}^{0}(M) \longrightarrow \mathrm{E}^{2} \mathrm{D}(M) \longrightarrow 0
$$

tells us that $\mathrm{E}^{1}\left(M / \operatorname{tor}_{\Lambda} M\right)$ fits into the exact sequence

$$
\mathrm{E}^{1} \mathrm{E}^{0} \mathrm{E}^{0}(M) \longrightarrow \mathrm{E}^{1}\left(M / \operatorname{tor}_{\Lambda} M\right) \longrightarrow \mathrm{E}^{2} \mathrm{E}^{2} \mathrm{D}(M),
$$

i.e. it suffices to show that $\mathrm{E}^{1} \mathrm{E}^{0} \mathrm{E}^{0}(M)$ is pseudo-null. But $\mathrm{E}^{1} \mathrm{E}^{1} \mathrm{E}^{0} \mathrm{E}^{0}(M)=0$ by 3.5 , (v), (and $\mathrm{E}^{0} \mathrm{E}^{1} \mathrm{E}^{0} \mathrm{E}^{0}(M)=0$ anyway), i.e. $j\left(\mathrm{E}^{1} \mathrm{E}^{0} \mathrm{E}^{0}(M)\right) \geq 2$ respectively $\delta\left(\mathrm{E}^{1} \mathrm{E}^{0} \mathrm{E}^{0}(M)\right) \leq d-2$.

\subsection{The graded ring $\operatorname{gr}(\Lambda)$}

An important method to verify the Auslander condition of a ring $\Lambda$ consists of endowing $\Lambda$ with a suitable filtration and studying the associated graded ring $\operatorname{gr}(\Lambda)$. By a filtration on a ring $\Lambda$ we mean an increasing (!) sequence of additive subgroups $\Sigma_{i-1} \subseteq \Sigma_{i} \subseteq \Sigma_{i+1}$ satisfying $\bigcup \Sigma_{i}=\Lambda$ and $\bigcap \Sigma_{i}=0$ and the inclusions $\Sigma_{i} \Sigma_{k} \subseteq \Sigma_{i+k}$ hold for all pairs of integers $i$ and $k$. The main example on a local ring is the $\mathfrak{M}$-adic filtration with $\Sigma_{-i}=\mathfrak{M}^{i}$ for all $i \geq 0$ (by convention, $\mathfrak{M}^{0}=\Lambda$ ). For our aim the closure condition will be crucial:

Definition 3.19. The filtration $\Sigma$ satisfies the closure condition if the additive subgroups $\Sigma_{m_{1}} u_{1}+\cdots+\Sigma_{m_{s}} u_{s}$ and $u_{1} \Sigma_{m_{1}}+\cdots+u_{s} \Sigma_{m_{s}}$ are closed with respect to the topology induced by $\Sigma$ for any finite subset $u_{1}, \ldots, u_{s}$ in $\Lambda$ and all integers $m_{1}, \ldots, m_{s}$. 
Lemma 3.20. Let $G$ be a $p$-adic analytic pro-p-group. Then the $\mathfrak{M}$-adic filtration on $\Lambda(G)$ satisfies the closure condition.

Proof. Note that the $\mathfrak{M}$-adic topology on $\Lambda$ coincides with the $(\mathfrak{m}, I)$-topology (cf. [27, (5.2.15)]). Since $\mathfrak{M}$ is a two-sided ideal of $\Lambda$ the subgroup $\mathfrak{M}^{i-m_{1}} u_{1}+$ $\cdots+\mathfrak{M}^{i-m_{s}} u_{s}, u_{1} \mathfrak{M}^{i-m_{1}}+\cdots+u_{s} \mathfrak{M}^{i-m_{s}}$ is a finitely generated left, right ideal, respectively. Hence, these subgroups are compact as continuous images of the compact module $\Lambda^{n}$ for some $n$.

Put $\operatorname{gr}(\Lambda)=\bigoplus \Sigma_{i} / \Sigma_{i-1}$, which is called the associated graded $\operatorname{ring}$ of $\Lambda$ with respect to the filtration $\Sigma$. The above lemma admits applying the following theorem of Björk to certain completed group rings:

\section{Theorem 3.21 (Björk).}

(i) Assume that $\operatorname{gr}(\Lambda)$ is a Auslander regular ring and that $\Sigma$ satisfies the closure condition. Then $\Lambda$ is a Auslander regular ring.

(ii) In the situation of (i), the equality $j(M)=j(\operatorname{gr}(M))$ holds. If, in addition, $\operatorname{gr}(\Lambda)$ is commutative and of pure dimension d, then also $\delta(M)=\operatorname{dim}(\operatorname{gr}(M))$ holds, where $\operatorname{dim}(\operatorname{gr}(M))=\operatorname{dim}\left(\operatorname{supp}_{\operatorname{gr}(\Lambda)}(\operatorname{gr}(M))\right)$ is the Krull dimension of $\operatorname{gr}(M)$.

Proof. See [4, Theorems 4.1,4.3] and also [5, Theorem 3.9. and Remark]. For the last equality note that

$$
\begin{aligned}
\operatorname{dim}(\operatorname{gr}(M)) & =\max \left\{\operatorname{dim}\left(\operatorname{gr}(M)_{\mathfrak{p}} \mid \mathfrak{p} \text { maximal ideal of } \operatorname{gr}(\Lambda)\right\}\right. \\
& =d-\min \left\{j\left(\operatorname{gr}(M)_{\mathfrak{p}}\right) \mid \mathfrak{p} \text { maximal ideal of } \operatorname{gr}(\Lambda)\right\} \\
& =d-j(\operatorname{gr}(M) \\
& =d-j(M) \\
& =\delta(M),
\end{aligned}
$$

where we used Proposition 3.5, (ii), and the fact that localization commutes with Ext-groups, if all objects are Noetherian.

Our task will be to determine the structure of $\operatorname{gr}(\Lambda(G))$. Before stating the next, result we recall that a pro- $p$-group $G$ is called extra-powerful, if the relation $[G, G] \subseteq G^{p^{2}}$ holds. Furthermore, note that $\operatorname{gr}\left(\mathbb{Z}_{p}\right) \cong \mathbb{F}_{p}\left[X_{0}\right]$ if $\mathbb{Z}_{p}$ is endowed with the $\mathfrak{m}$-adic filtration.

Theorem 3.22. Let $G$ be a uniform and extra-powerful pro-p-group of dimension $\operatorname{dim}(G)=r$. Then there is a $\operatorname{gr}\left(\mathbb{Z}_{p}\right)$-algebra-isomorphism

$$
\operatorname{gr}(\Lambda(G)) \cong \mathbb{F}_{p}\left[X_{0}, \ldots, X_{r}\right] .
$$

In particular, $\operatorname{gr}(\Lambda(G))$ is a commutative regular Noetherian ring.

A consequence of Lazard's results is the

Remark 3.23. Any compact $p$-adic analytic group contains an open characteristic subgroup, which is an uniform and extrapowerful pro- $p$-group (cf. [12, Corollary 9.36] and [37, Proposition 8.5.3]). 
For the proof of the theorem we need some more terminology. Let $G$ be an uniform pro- $p$-group with a minimal system of (topological) generators $\left\{x_{1}, \ldots, x_{r}\right\}$, in particular $\operatorname{dim}(G)=r$. Then the lower $p$-series is given by $P_{1}(G)=G, P_{i+1}(G)$ $=\left(P_{i}(G)\right)^{p}, i \geq 1$. This filtration defines a $p$-valuation $\omega: G \longrightarrow \mathbb{N}_{>0} \cup\{\infty\} \subseteq$ $\mathbb{R}_{>0} \cup\{\infty\}$ of $G$ in the sense of Lazard via $\omega(g):=\sup \left\{i \mid g \in P_{i}(G)\right\}$, which induces a filtration on the group algebra $\mathbb{Z}_{p}[G]$ of the underlying abstract group of $G$, too (cf. [25, Chap. III, 2.3.1.2]).

Lemma 3.24. The filtration on $\mathbb{Z}_{p}[G]$, induced by $\omega$, is the $\mathfrak{M}_{d}$-adic one, where $\mathfrak{M}_{d}=\mathfrak{m}+I_{d}(G)$ with the augmentation ideal $I_{d}(G)$ of $\mathbb{Z}_{p}[G]$.

Proof. Conferring the proof of Lemma III, (2.3.6) in [25], the induced filtration is given by the following ideals in $\mathbb{Z}_{p}[G], n \in \mathbb{N}: A_{n}$ is generated as $\mathbb{Z}_{p}$-module by the elements $p^{l}\left(g_{1}-1\right) \cdots\left(g_{m}-1\right)$ where $l, m \in \mathbb{N}, g_{i} \in G$ and $l+\omega\left(g_{1}\right)+\ldots+\omega\left(g_{m}\right)$ $\geq n$, whereas the $\mathfrak{M}_{d}$-adic filtration is defined by the ideals $\mathfrak{M}_{d}^{n}$, which are generated (over $\left.\mathbb{Z}_{p}[G]\right)$ by the elements $p^{l}\left(g_{1}-1\right) \cdots\left(g_{m}-1\right)$, where $l, m \in \mathbb{N}, g_{i} \in G$ and $l+m=n$. Since $\omega(g) \geq 1$ for all $g \in G$ the ideal $\mathfrak{M}_{d}^{n}$ is contained in $A_{n}$. The converse is a consequence of the following

Claim. Let $g \in G$ with $\omega(g)=t \geq 1$, then $g-1 \in \mathfrak{M}_{d}^{t}$.

Since $G$ is uniform, the map $G \longrightarrow P_{t}(G)$, which assigns $g p^{t-1}$ to $g$, is surjective (cf. [12, Lemma 4.10]), i.e. there exists an element $h \in G$ with $g=h^{p^{t-1}}$. Writing

$$
g-1=(1+(h-1))^{p^{t-1}}-1=\sum_{k \geq 1}\left(\begin{array}{c}
p^{t-1} \\
k
\end{array}\right)(h-1)^{k},
$$

one verifies that $g-1 \epsilon \mathfrak{M}_{d}^{t}$, because $v_{p}\left(\left(\begin{array}{c}p^{t-1} \\ k\end{array}\right)\right)=t-1-v_{p}(k) \geq t-k$, i.e. $\left(\begin{array}{c}p^{t-1} \\ k\end{array}\right)(h-1)^{k} \in \mathfrak{M}_{d}^{t}$.

Lemma 3.25. The $\mathfrak{M}_{d}$-adic filtration on $\mathbb{Z}_{p}[G]$ induces the $\mathfrak{M}$-adic filtration on $\mathbb{Z}_{p} \llbracket G \rrbracket$.

Proof. The ideals defining the induced filtration are just the closure $\overline{\mathfrak{M}_{d}^{n}}$ of $\mathfrak{M}_{d}^{n} \subseteq$ $\mathbb{Z}_{p}[G] \subseteq \mathbb{Z}_{p} \llbracket G \rrbracket$ with respect to the $\mathfrak{M}$-adic topology on $\mathbb{Z}_{p} \llbracket G \rrbracket$. Since they contain the elements $p^{l}\left(g_{1}-1\right) \cdots\left(g_{m}-1\right)$ with $l, m \in \mathbb{N}, g_{i} \in\left\{x_{1}, \ldots, x_{r}\right\}$ and $l+m=n$, which generate $\mathfrak{M}^{n}$ as ideal of $\mathbb{Z}_{p} \llbracket G \rrbracket$, they contain $\mathfrak{M}^{n}$, too. On the other hand $\mathfrak{M}^{n}$ is closed and contains all the generators of the $\mathbb{Z}_{p}[G]$-ideal $\mathfrak{M}_{d}^{n}$ : $p^{l}\left(g_{1}-1\right) \ldots\left(g_{m}-1\right), l, m \in \mathbb{N}, g_{i} \in G$. This proves the lemma.

Now we can prove Theorem 3.22.

Proof. Since $\operatorname{gr}(G)=\bigoplus P_{i}(G) / P_{i+1}(G)$ is a Lie algebra, which is free of rank $r$ as $\operatorname{gr}\left(\mathbb{Z}_{p}\right)$-module, we get the following inclusion:

$$
\begin{aligned}
\operatorname{gr}(G) \subseteq U g r(G) & \cong \operatorname{gr}\left(\mathbb{Z}_{p}[G]\right) \\
& \cong \operatorname{gr}\left(\mathbb{Z}_{p} \llbracket G \rrbracket\right),
\end{aligned}
$$


where the first equation holds by [25, Chap. III, 2.3.3] and $\operatorname{Ugr}(G)$ is the enveloping algebra of the Lie algebra $\operatorname{gr}(G)$, whereas the second one is a consequence of Lemma 3.25. According to [37, Theorem 8.7.7], the graded $\operatorname{ring} \operatorname{gr}\left(\mathbb{Z}_{p} \llbracket G \rrbracket\right)$ is commutative ( $G$ is assumed to be extra-powerful), i.e.

$$
\operatorname{Ugr}(G) \cong \operatorname{gr}\left(\mathbb{Z}_{p}\right)\left[X_{1}, \ldots, X_{r}\right] \cong \mathbb{F}_{p}\left[X_{0}, \ldots, X_{r}\right]
$$

As an important consequence we obtain the

Theorem 3.26. Let $G$ be a compact p-adic analytic group without p-torsion. Then the completed group ring $\Lambda(G)$ is an Auslander regular ring.

Proof. $G$ posses an open characteristic subgroup $N$ which is an uniform, extrapowerful pro- $p$-group. By the theorem of Björk and Theorem 3.22, $\Lambda(N)$ is an Auslander regular ring, because $\operatorname{gr}\left(\mathbb{Z}_{p} \llbracket N \rrbracket\right)$ has this property as a regular commutative Noetherian ring (cf. [3, pp.65-69]). But $\mathrm{E}_{\Lambda(G)}^{i}(M) \cong \mathrm{E}_{\Lambda(N)}^{i}(M)$ as $\Lambda(N)$-modules for any $\Lambda(G)$-module $M$, by which the Auslander condition is easily verified.

If one is not interested in the $\mathbb{Z}_{p}$-torsion submodule of a $\Lambda$-module $M$ it might be convenient to replace $M$ by its maximal $\mathbb{Z}_{p}$-torsion-free quotient $M /$ tor $_{\mathbb{Z}_{p}} M$. This leads to the study of the subcategory $\Lambda-\bmod ^{f l}$ of $\Lambda-\bmod$ consisting of $\mathbb{Z}_{p^{-}}$ torsionfree or equivalently $\mathbb{Z}_{p}$-flat $\Lambda$-modules. This category is closely related to the category $\mathbb{Q}_{p} \llbracket G \rrbracket-$ mod of finitely generated $\mathbb{Q}_{p} \llbracket G \rrbracket$-modules where

$$
\mathbb{Q}_{p} \llbracket G \rrbracket:=\mathbb{Z}_{p} \llbracket G \rrbracket \otimes_{\mathrm{Z}_{p}} \mathbb{Q}_{p}
$$

by definition. Note that the latter ring is Noetherian whenever $\mathbb{Z}_{p} \llbracket G \rrbracket$ is. We let $\Lambda-\bmod _{\mathbb{Q}_{p}}^{\mathrm{fl}}$ denote the category with the same objects as $\Lambda-\bmod ^{\mathrm{fl}}$ and such that

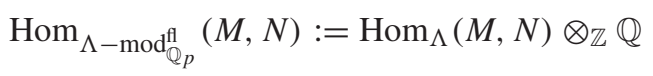

for any two $\Lambda$-modules $M, N$ in $\Lambda-\bmod ^{\mathrm{fl}}$ with the composition of homomorphisms being the $\mathbb{Q}$-linear extension of the composition in $\Lambda-\bmod ^{\mathrm{fl}}$. It is clear that the functor $M \mapsto M_{\mathbb{Q}_{p}}:=M \otimes_{\mathbb{Z}_{p}} \mathbb{Q}_{p}$ induces an equivalence of categories

$$
\Lambda-\bmod _{\mathbb{Q}_{p}}^{\mathrm{fl}} \cong \mathbb{Q}_{p} \llbracket G \rrbracket-\bmod
$$

Remark 3.27. Schneider and Teitelbaum proved in [32] that, for a compact $p$-adic Lie group, these categories are anti-equivalent to the category of certain Banach space representations of $G$.

The following proposition, which is standard, allows to calculate Ext-groups in $\mathbb{Q}_{p} \llbracket G \rrbracket-\bmod$ via Ext-groups in $\Lambda$-mod.

Proposition 3.28. For any $M, N$ in $\Lambda$-mod we have canonical isomorphisms

$$
\operatorname{Ext}_{\Lambda}^{i}(M, N) \otimes_{\mathbb{Z}_{p}} \mathbb{Q}_{p} \cong \operatorname{Ext}_{\mathbb{Q}_{p} \llbracket G \rrbracket}^{i}\left(M_{\mathbb{Q}_{p}}, N_{\mathbb{Q}_{p}}\right) \text { for all } i \geq 0 \text {. }
$$


Now, we are able to derive the Auslander regularity of $\mathbb{Q}_{p} \llbracket G \rrbracket$ from the Auslander regularity of $\Lambda(G)$.

Theorem 3.29. Let $G$ be a compact p-adic analytic group without p-torsion. Then the group ring $\mathbb{Q}_{p} \llbracket G \rrbracket$ is an Auslander regular ring of (projective) dimension $\operatorname{pd}_{p} \llbracket G \rrbracket=\operatorname{cd}_{p}(G)$.

Proof. We set $r:=\operatorname{cd}_{p} G$. Let $M$ be any finitely generated $\mathbb{Q}_{p} \llbracket G \rrbracket$-module and choose $N \in \Lambda-\bmod ^{\mathrm{fl}}$ with $M \cong N_{\mathbb{Q}_{p}}$. Since $N$ is $\mathbb{Z}_{p}$-torsionfree, we have $E_{\Lambda}^{r+1}(N)=0$ by Theorem 4.7 (iii), hence $\operatorname{pd}_{\Lambda} N \leq d$ using (6.3). By the previous proposition, $\mathrm{E}_{\mathbb{Q}_{p} \llbracket G \rrbracket}^{r+1}(M)$ vanishes, too, i.e. $\operatorname{pd}_{\mathbb{Q}_{p} \llbracket G \rrbracket} M \leq r$. On the other hand the projective dimension of $\mathbb{Q}_{p}$ is $r$, because $\mathrm{E}_{\mathbb{Q}_{p} \llbracket G \rrbracket}^{r}\left(\mathbb{Q}_{p}\right)=\mathrm{E}^{r}\left(\mathbb{Z}_{p}\right) \otimes_{\mathbb{Z}_{p}} \mathbb{Q}_{p} \cong \mathbb{Q}_{p}$ by $[22,2.6]$. It follows that $\operatorname{pd} \mathbb{Q}_{p} \llbracket G \rrbracket=r$.

Now we will verify the Auslander condition: Since any $\mathbb{Q}_{p} \llbracket G \rrbracket$-submodule of $\mathrm{E}_{\mathbb{Q}_{p} \llbracket G \rrbracket}^{i}(M) \cong \mathrm{E}_{\Lambda}^{i}(N) \otimes_{\mathbb{Z}_{p}} \mathbb{Q}_{p}$ has the form $L_{\mathbb{Q}_{p}}$ for some $\Lambda$-submodule $L \subseteq$ $\mathrm{E}_{\Lambda}^{i}(N)$ we see that

$$
\mathrm{E}_{\mathbb{Q}_{p} \llbracket G \rrbracket}^{j}\left(L_{\mathbb{Q}_{p}}\right) \cong \mathrm{E}_{\Lambda}^{j}(L) \otimes_{\mathbb{Z}_{p}} \mathbb{Q}_{p}=0, j<i,
$$

because $\Lambda$ is Auslander regular by Theorem 3.26.

\subsection{The $\mu$-invariant}

For the purpose to study the $p$-torsion part tor $_{\mathbb{Z}_{p}} M$ of a $\Lambda$-module $M$ we are also interested in the rings $\mathbb{Z} / p^{m} \llbracket G \rrbracket \cong \Lambda(G) / p^{m}$, especially the ring $\mathbb{F}_{p} \llbracket G \rrbracket$, and will consider the change of rings $\Lambda(G) \rightarrow \Lambda / p^{m}$. For a $\Lambda / p^{m}$-module $M$ there exists a convergent spectral sequence (see [36, Example 5.6.3])

$$
\operatorname{Ext}_{\Lambda / p^{m}}^{i}\left(M, \operatorname{Ext}_{\Lambda}^{j}\left(\Lambda / p^{m}, \Lambda\right)\right) \Rightarrow \operatorname{Ext}_{\Lambda}^{i+j}(M, \Lambda)
$$

We should mention that here $\operatorname{Ext}_{\Lambda}^{j}\left(\Lambda / p^{m}, \Lambda\right)$ is a left $\Lambda$ - and $\Lambda / p^{m}$-module by functoriality and the right $\Lambda$-structure of the bi-module $\Lambda / p^{m}$. Using the free resolution

$$
0 \longrightarrow \Lambda \stackrel{p^{m}}{\longrightarrow} \Lambda \longrightarrow \Lambda / p^{m} \longrightarrow 0
$$

it is easy to calculate that

$$
\operatorname{Ext}_{\Lambda}^{j}\left(\Lambda / p^{m}, \Lambda\right) \cong \begin{cases}\Lambda / p^{m} & \text { if } j=1 \\ 0 & \text { otherwise }\end{cases}
$$

Hence the spectral sequence degenerates to

$$
\mathrm{E}_{\Lambda / p^{m}}^{i}(M) \cong \mathrm{E}_{\Lambda}^{i+1}(M)
$$

for any $\Lambda / p^{m}$-module $M$ and any integer $i$. We obtain the following 
Theorem 3.30. Let $G$ be a compact p-adic analytic group without p-torsion and $m$ any natural number. Then

(i) $\mathbb{Z}_{p} / p^{m} \llbracket G \rrbracket$ is an Auslander-Gorenstein ring of injective dimension $\mathrm{cd}_{p}(G)$.

(ii) $\mathbb{F}_{p} \llbracket G \rrbracket$ is an Auslander regular ring of dimension $\operatorname{cd}_{p}(G)$.

Remark 3.31. The same arguments prove that $\Lambda(G) /(f)$ is a Auslander Gorenstein ring of injective dimension $\operatorname{cd}_{p}(G)$ for any element $f$ of the center of $\Lambda(G)$ or even more general for any $f \in \Lambda(G)$ such that the left ideal $(f):=\Lambda(G) f$ is two-sided.

Proof. From the above formula we derive that $\Lambda / p^{m}$ has finite injective dimension $\operatorname{cd}_{p}(G)$. On the other hand it is well known that the projective dimension of $\mathbb{F}_{p} \llbracket G \rrbracket$ is equal to $\operatorname{cd}_{p}(G)$ (see [27, V§2, Example 5]). Hence it suffices to verify the Auslander condition: For a $\Lambda / p^{m}$-module $M$ let $N \subseteq \mathrm{E}_{\Lambda / p^{m}}^{i}(M)$ be a $\Lambda / p^{m}$ submodule which we will also consider as $\Lambda$-submodule of $\mathrm{E}_{\Lambda}^{i+1}(M)$. Applying again the above isomorphism, we see that $\mathrm{E}_{\Lambda / P^{m}}^{j}(N) \cong \mathrm{E}_{\Lambda}^{j+1}(N)=0$ for any integer $j<i$ because $\Lambda$ fulfills the Auslander condition.

A different possibility to prove (ii) of the previous theorem would be to imitate the proof of Theorem 3.26 using the analogue of Theorem 3.22: if $G$ is a uniform pro- $p$-group of dimension $d$, then there is an isomorphism

$$
\operatorname{gr}\left(\mathbb{F}_{p} \llbracket G \rrbracket\right) \cong \mathbb{F}_{p}\left[X_{1}, \ldots, X_{r}\right],
$$

where $\mathbb{F}_{p} \llbracket G \rrbracket$ is endowed with its $\mathfrak{M}$-adic filtration (see [37, 8.7.10]). In particular, $\mathbb{F}_{p} \llbracket G \rrbracket$ has no zero divisors for uniform $G([37,8.7 .9])$.

In order to measure the size of the $p$-torsion part of a $\Lambda$-module we have (as usual) the $\mu$-invariant which is defined as follows.

Definition 3.32. Assume that $G$ is a $p$-adic Lie group without $p$-torsion such that $\mathbb{F}_{p} \llbracket G \rrbracket$ is integral. For any $\Lambda(G)$-module $M$ we define its $\mu$-invariant $\mu(M)$ as

$$
\mu(M)=\operatorname{rk}_{\mathbb{F}_{p} \llbracket G \rrbracket} \bigoplus_{i \geq 0} p^{i+1} M / p^{i} M,
$$

where $p_{p^{0}} M=0$ by convention. Observe that the sum is finite because $\Lambda$ is Noetherian.

Susan Howson has defined the $\mu$-invariant in a similar, equivalent way and she has independently studied its properties in [19]. In particular, she expresses the $\mu$-invariant of a $\Lambda$-module $M$ to the Euler characteristic of tor $\mathbb{Z}_{p} M$.

Note that the $\mu$-invariant only depends on the $\Lambda$-resp. $\mathbb{Z}_{p}$-torsion-submodule: $\mu(M)=\mu\left(\operatorname{tor}_{\Lambda} M\right)=\mu\left(\operatorname{tor}_{\mathbb{Z}_{p}} M\right)=\mu\left(p^{m} M\right)$ for $m$ sufficiently large. With respect to the vanishing we have the following characterization:

Remark 3.33. Since $p^{i+1} M / p^{i} M \stackrel{p^{i}}{\longrightarrow}{ }_{p} M$ the following is equivalent

$$
\begin{aligned}
\mu(M)=0 & \Leftrightarrow \mu\left({ }_{p} M\right)=0 \\
& \Leftrightarrow{ }_{p} M \text { is } \mathbb{F}_{p} \llbracket G \rrbracket \text {-torsion } \\
& \Leftrightarrow{ }_{p} M \text { is a pseudo-null } \Lambda \text {-module. }
\end{aligned}
$$

For the latter equivalence we used again the above isomorphism. 
The next proposition shows that the $\mu$-invariant is in fact an invariant "up to pseudo-isomorphism", i.e. it factors through the quotient category $\Lambda$-mod $/ \mathcal{P} \mathcal{N}$. In particular, our definition of $\mu$ generalizes the usual definition via the structure theory if $G$ is isomorphic to $\mathbb{Z}_{p}^{r}$ for some $r$.

Proposition 3.34. Let $G$ be a p-adic analytic group without $p$-torsion such that both $\Lambda=\Lambda(G)$ and $\Lambda / p$ are integral. Then

$$
M \sim N \text { implies } \mu(M)=\mu(N) .
$$

Proof. The statement will follow if it holds in the two special cases of exact sequences

(a) $0 \longrightarrow Q \longrightarrow N \longrightarrow N \longrightarrow 0$,

(b) $0 \longrightarrow M \longrightarrow N \longrightarrow Q \longrightarrow 0$,

where $Q$ is pseudo-null. More generally, we consider a short exact sequence of $\Lambda$-modules

$$
0 \longrightarrow X \longrightarrow Y \longrightarrow Z \longrightarrow 0 \text {. }
$$

The snake lemma implies the exactness and commutativity of the following diagram

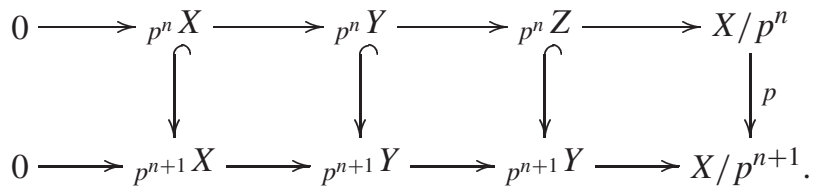

Again by the snake lemma we obtain the exact sequences

$$
\begin{gathered}
0 \longrightarrow p^{n+1} X / p^{n} X \longrightarrow p^{n+1} Y / p^{n} Y \longrightarrow A_{n+1} / A_{n} \longrightarrow 0, \\
0 \longrightarrow K_{n} \longrightarrow A_{n+1} / A_{n} \longrightarrow p^{n+1} Z / p^{n} Z \longrightarrow B_{n+1} / B_{n} \longrightarrow 0
\end{gathered}
$$

where $A_{i}$ denotes the image of ${ }_{p^{i}} Y$ in $p_{p^{i}} Z$ with cokernel $B_{i}$, the latter module considered as submodule of $X / p^{i}$, and $K_{n}:=\operatorname{ker}\left(B_{n} \rightarrow B_{n+1}\right)$. In case (b) $A_{n+1} / A_{n}$ is a pseudo-null $\Lambda$-module because $A_{n+1} \subseteq Z$. Hence $\operatorname{rk}_{\mathbb{F}_{p} \llbracket G \rrbracket} A_{n+1} / A_{n}=0$ by

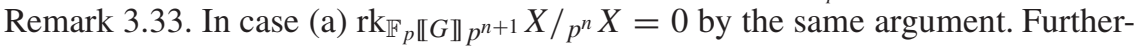
more, $K_{n} \subseteq X / p^{n}, B_{n+1} \subseteq X / p^{n+1}$ and finally $B_{n+1} / B_{n}$ are pseudo-null, too.

By $\Lambda-\bmod (p)$ we shall write the plain subcategory of $\Lambda$-mod consisting of $\mathbb{Z}_{p}$-torsion modules while by $\mathcal{P N}(p)$ " $=\mathcal{P} \mathcal{N} \cap \Lambda-\bmod (p)$ " we denote the Serre subcategory of $\Lambda-\bmod (p)$ the objects of which are pseudo-null $\Lambda$-modules. In other words $M$ belongs to $\mathcal{P N}(p)$ if and only if it is a $\Lambda / p^{n}$-module for an appropriate $n$ such that $\mathrm{E}_{\Lambda / p^{n}}^{0}(M)=0$. Recall that there is a canonical exact functor $q: \Lambda-\bmod (p) \rightarrow \Lambda-\bmod (p) / \mathcal{P N}(p)$. For the description of the $p$-torsion part the following result will be crucial. 
Proposition 3.35. Assume that $G$ is a $p$-adic analytic group without $p$-torsion such that both $\Lambda=\Lambda(G)$ and $\Lambda / p$ are integral. Then the following holds:

(i) $q(\Lambda / p)$ is simple object in $\Lambda-\bmod (p) / \mathcal{P N}(p)$, i.e. does not contain any proper subobject.

(ii) Every object $A$ in $\Lambda-\bmod (p) / \mathcal{P N}(p)$ has a finite composition series

$$
0=A_{0} \subseteq A_{1} \subseteq \cdots \subseteq A_{i+1}=A
$$

of subobjects $A_{j}$ of $A$ such that $A_{j+1} / A_{j} \cong q(\Lambda / p)$ for every $i \geq j \geq 0$. In particular, $q(\Lambda / p)$ is the unique simple object of $\Lambda-\bmod (p) / \mathcal{P N}(p)$.

(iii) Any $q(M)$ in $\Lambda-\bmod (p) / \mathcal{P N}(p)$ has finite length equal to $\mu(M)$. Thus, $[q(M)] \mapsto \mu(M)$ induces an isomorphism

$$
K_{0}(\Lambda-\bmod (p) / \mathcal{P} \mathcal{N}(p)) \cong \mathbb{Z}
$$

We need the following lemma which can be proved literally as [18, Lemma 2.25] because $\mathbb{F}_{p} \llbracket G \rrbracket$ is both left and right Noetherian ring without zero divisors and thus it has a skew field of fractions.

Lemma 3.36. With the assumptions of the proposition let $M$ be a torsion-free $\Lambda / p$-module of rank $\mathrm{rk}_{\Lambda / p}(M)=m$. Then there exist free $\Lambda / p$-modules $F, F^{\prime}$ such that $F \subseteq M, M \subseteq F^{\prime}$ and both $M / F$ and $F^{\prime} / M$ are $\Lambda / p$-torsion, i.e. pseudonull considered as $\Lambda$-module. In particular, for any $\Lambda / p$-module of rank $m$ there is an isomorphism

$$
q(M) \cong q(\Lambda / p)^{m}
$$

Proof. Let $h: q(M) \hookrightarrow q(\Lambda / p)$ be a monomorphism in the quotient category. By [33, I 2.9] there exists a diagram

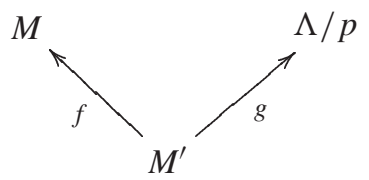

in $\Lambda-\bmod (p)$ with $f$ a pseudo-isomorphism in $\Lambda-\bmod$ such that

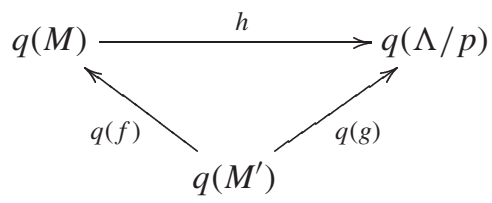

commutes. Since $h$ is a monomorphism and $q(f)$ an isomorphism, $\operatorname{ker}(g)$ must be in $\mathcal{P} \mathcal{N}(p)$. Since $M^{\prime} / \operatorname{ker}(g) \subseteq \Lambda / p$, we can consider its $\Lambda / p$-rank which can be either 1 or 0 . In the first case we conclude that $g$ is a pseudo-isomorphism, i.e. $q(g)$ is an isomorphism, while in the second case $M^{\prime} / \operatorname{ker}(g)$ and hence $M^{\prime}$ is pseudo-null, thus $q\left(M^{\prime}\right)=0$. This proves (i). 
For any $M \epsilon \Lambda-\bmod (p)$, the canonical decomposition

$$
0 \subseteq{ }_{p} M \subseteq p^{2} M \subseteq \cdots \subseteq p^{m} M=M
$$

for some $m$, induces a decomposition

$$
0 \subseteq q\left({ }_{p} M\right) \subseteq q\left(p_{p^{2}} M\right) \subseteq \cdots \subseteq q\left(p^{m} M\right)=q(M)
$$

with

$$
q\left({ }_{p^{j+1}} M\right) / q\left({ }_{p^{j}} M\right) \cong q\left({ }_{p^{j+1}} M / p_{p^{j}} M\right) \cong q(\Lambda / p)^{d_{j}},
$$

where $d_{j}=\operatorname{rk}_{\Lambda / p}\left(p^{j+1} M / p^{j} M\right)$ by the previous lemma. Since this filtration can be refined easily to a decomposition series of the desired kind, we are done.

Corollary 3.37. The invariant $\mu$ is additive on short exact sequences of $\Lambda$-torsion modules.

Proof. Since $\mu$ is additive on short exact sequences of $p$-torsion modules by the proposition it suffices to reduce the general statement to this case. Let

$$
0 \longrightarrow X \longrightarrow Y \longrightarrow O
$$

be a short exact sequence of $\Lambda$-torsion modules. Choosing a number $n$ such that the $p$-torsion parts of $X, Y$ and $Z$ are annihilated by $p^{n}$, we obtain an exact sequence

$$
0 \longrightarrow \operatorname{tor}_{\mathbb{Z}_{p}} X \longrightarrow \operatorname{tor}_{\mathbb{Z}_{p}} Y \longrightarrow \operatorname{tor}_{\mathbb{Z}_{p}} Z \longrightarrow X / p^{n} \stackrel{\varphi}{\longrightarrow} Y / p^{n} \text {. }
$$

Considering the exact, commutative diagram

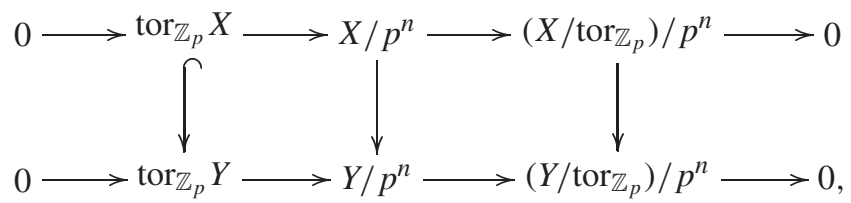

we see that $\operatorname{ker}(\varphi)$ is pseudo-null by the following lemma, i.e.

$$
0 \longrightarrow \operatorname{tor}_{\mathbb{Z}_{p}} X \longrightarrow \operatorname{tor}_{\mathbb{Z}_{p}} Y \longrightarrow \operatorname{tor}_{\mathbb{Z}_{p}} Z \longrightarrow 0
$$

is exact $\bmod \mathcal{P} \mathcal{N}$.

Lemma 3.38. Assume that $G$ is a p-adic analytic group without p-torsion such that both $\Lambda=\Lambda(G)$ and $\Lambda / p$ are integral. Let $M$ be a (not necessarily torsion) $\Lambda$-module. Then the following holds

$$
\mu(M / p)=0 \Rightarrow \mu\left({ }_{p} M\right)=0 .
$$

Proof. Since $\left(\operatorname{tor}_{\mathbb{Z}_{p}} M\right) / p \subseteq M / p$ by the snake lemma, it suffices to deal with the case that $M$ is $\Lambda$-torsion. But then the additivity of the $\mu$-invariant shows immediately that $\mu\left({ }_{p} M\right)=\mu(M / p)$. 
Lemma 3.39. Assume that $G$ is a p-adic analytic group without p-torsion such that both $\Lambda=\Lambda(G)$ and $\Lambda / p$ are integral. Let $M$ be a $\Lambda$-torsion module with tor $_{\mathbb{Z}_{p}} M=0$. Then

(i) for any integer $n \geq 1$, the module $M / p^{n}$ is pseudo-null.

(ii) $\operatorname{tor}_{\mathbb{Z}_{p}} \mathrm{E}^{1}(M)=0$.

We will denote the annihilator in $\Lambda$ of an element $m \in M$ by $a n n_{\Lambda}(m):=$ $\{\lambda \in \Lambda \mid \lambda m=0\}$.

Proof. Since there is a surjection

$$
\bigoplus \Lambda / a n n_{\Lambda}\left(m_{i}\right) \rightarrow M
$$

for a finite set of generators $m_{i}$ of $M$, it suffices to prove (i) in the case $M:=\Lambda / I$, where $I$ is a non-zero left ideal of $\Lambda$. As $M / p^{n}$ is $\Lambda$-torsion we are done once we have shown the vanishing of $\mathrm{E}_{\Lambda}^{1}\left(M / p^{n}\right)$. But

$$
\begin{aligned}
\mathrm{E}_{\Lambda}^{1}\left(M / p^{n}\right) & \cong \mathrm{E}_{\Lambda / p^{n}}^{0}\left(M / p^{n}\right) \\
& \cong \operatorname{Hom}_{\Lambda / p^{n}}\left(M, \Lambda / p^{n}\right) \\
& \cong \operatorname{Hom}_{\Lambda}\left(\Lambda / I, \Lambda / p^{n}\right)=0 .
\end{aligned}
$$

Indeed, the vanishing of the latter module can be seen as follows: let $\varphi: \Lambda \rightarrow \Lambda / p^{n}$ be a non-trivial homomorphism of $\Lambda$-modules which factors through $\Lambda / I$, i.e. $I \subseteq \operatorname{ann}_{\Lambda}\left(x \bmod p^{n}\right)$ with $x \equiv \varphi(1) \bmod p^{n}$.

Claim. $\operatorname{ann}_{\Lambda}\left(x \bmod p^{n}\right) \subseteq p \Lambda$.

Let $\lambda \in \operatorname{ann}_{\Lambda}\left(x \bmod p^{n}\right)$, i.e. $\lambda x=p^{n} y$ for some $y \in \Lambda$ and let $n_{o}$ be the maximal integer with $x \in p^{n_{0}} \Lambda$, i.e. $x=p^{n_{0}} x_{0}$ for some $x_{0} \in \Lambda \backslash p \Lambda$ and $n_{0}<n$. Since the multiplication by $p^{n_{0}}$ is injective we obtain $\lambda x_{o}=p^{n-n_{0}} y \equiv 0 \bmod p$. Hence $\lambda \epsilon p \Lambda$ because $\Lambda / p$ is integral. This proves the claim.

The fact that ${ }_{p} M=0$, implies $I \cap p \Lambda=p I$ and regarding the claim it holds

$$
I=I \cap p \Lambda=p I=\ldots=p^{m} I
$$

for any $m \geq 0$. Since $p^{m}$ tends to zero if $m$ goes to infinity the ideal $I$ must be zero, a contradiction.

The second statement results from the first one regarding the exact sequence

$$
0=\mathrm{E}^{1}(M / p) \longrightarrow \mathrm{E}^{1}(M) \stackrel{p}{\longrightarrow} \mathrm{E}^{1}(M) .
$$

We finish this section with a "structure theorem for the $p$-torsion part of $\Lambda$ modules."

Theorem 3.40. Assume that $G$ is a p-adic analytic group without p-torsion such that both $\Lambda=\Lambda(G)$ and $\Lambda / p$ are integral. Let $M$ be in $\Lambda-\bmod (p)$. Then there exist uniquely determined natural numbers $n_{1}, \ldots, n_{r}$ and an isomorphism in $\Lambda-\bmod (p) / \mathcal{P} \mathcal{N}(p)$

$$
M \equiv \bigoplus_{1 \leq i \leq r} \Lambda / p^{n_{i}} \bmod \mathcal{P} \mathcal{N}(p)
$$


Proof. Let $m$ be minimal with the property: $p^{m+1} M=M$. The theorem is proved using induction with respect to $m$. The case $m=0$ is just Lemma 3.36, so let $m$ be arbitrary. Again by Lemma 3.36 we are in the following situation:

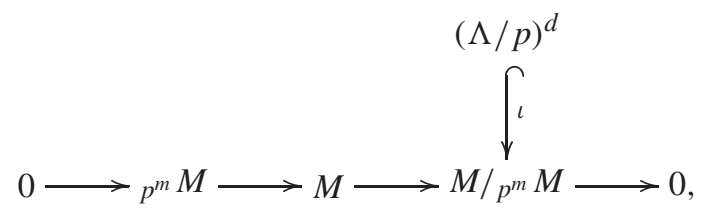

where $d$ is the $\Lambda / p$-rank of $M / p^{m} M$ and the cokernel of $\iota$ is pseudo-null. Replacing $M$ by the pull-back with $\iota$, we may assume that $M / p^{m} M \cong(\Lambda / p)^{d}$. Since $\left(\Lambda / p^{m+1}\right)^{d}$ is free in the category of $\Lambda / p^{m+1}$-modules, we obtain easily the following exact and commutative diagram

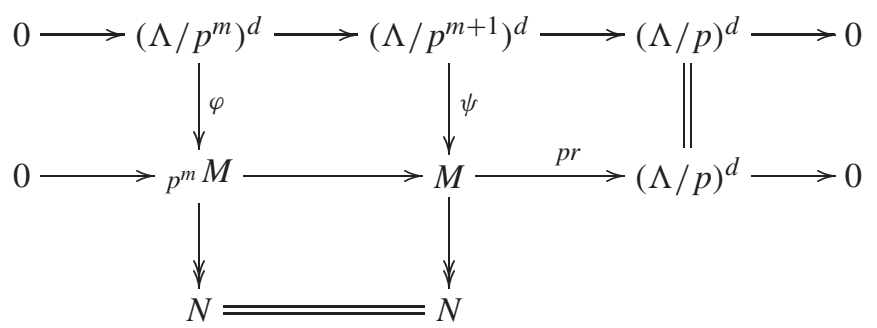

where $N$ is by definition the cokernel of $\psi$ respectively $\varphi$. First we will show that $\psi$ and hence also $\varphi$ is injective. Since $\left(\Lambda / p^{m+1}\right)^{d}$ - being of projective $\Lambda$-dimension 1 - does not contain any proper pseudo-null $\Lambda$-submodule, it suffices to prove that $\operatorname{ker}(\psi)$ is pseudo-null. Assuming the contrary, i.e. that $\mu(\operatorname{ker}(\psi)) \neq 0$, it follows that $\mu\left({ }_{p^{m+1}} K / p^{m} K\right)<d$ for the image $K$ of $\psi$ because for an arbitrary $p$-torsion $\Lambda$-module $N \operatorname{rk}_{\Lambda / p}\left(p^{i+1} N / p^{i} N\right) \geq \operatorname{rk}_{\Lambda / p}\left(p^{i+2} N / p^{i+1} N\right)$ holds for any $i \geq 0$. But this contradicts the surjectivity of $p r \circ \psi$.

To prove the theorem we only have to show that $\varphi$ has a co-section in $\Lambda-\bmod (p) / \mathcal{P N}(p)$, i.e. that the short exact sequence in the left column splits. Indeed, then a section $N \hookrightarrow p^{m} M$ would give rise to a section $N \hookrightarrow M$, i.e. $M \cong N \oplus\left(\Lambda / p^{m+1}\right)^{d}$, and by the assumption of the induction $N$ is already of the desired form. Here and in what follows we are arguing in the quotient category $\Lambda-\bmod (p) / \mathcal{P N}(p)$, though we omit the functor $q$ in the notation of maps and objects for simplicity.

Again by this assumption, the module $p^{m} M$ is isomorphic to a module of the form $\left(\Lambda / p^{m}\right)^{d^{\prime}} \oplus \bigoplus_{i} \Lambda / p^{n_{i}}$, where $n_{i}<m$. Assume first that $d=1$. We claim that the image of $\varphi$ is mapped surjectively onto one of the factors $\Lambda / p^{m}$ under the correspondent projection. Indeed, it is easy to see that otherwise the image would be contained in $p^{m-1} M$, which contradicts the injectivity of $\varphi$. Counting $\mu$-invariants, we see that $\varphi$ followed by the projection onto such a factor gives an isomorphism and therefore induces the desired co-section. If $d>1$ we make the same procedure iteratively for every factor of $\left(\Lambda / p^{m}\right)^{d}$ after first splitting up the 
image of the previous factor(s). The theorem follows because the uniqueness can be deduced easily from the decomposition

$$
0 \subseteq{ }_{p} M \subseteq p^{2} M \subseteq \cdots \subseteq p^{m} M=M
$$

counting $\Lambda / p$-ranks.

Remark 3.41. If one replaces $p \in \Lambda(G)$ by any element $f$ in the center of $\Lambda(G)$ such that $(f):=\Lambda(G) f$ is a prime ideal, i.e. such that $\Lambda(G) /(f)$ is integral, then one gets analogous results concerning the " $f$-torsion part" $\left\{m \in M \mid f^{n} m=0\right.$ for some $n$ \} of $M$. In particular, one obtains further invariants $\mu_{f}$ for these prime elements.

\section{Spectral sequences for Iwasawa adjoints}

In the previous section we have seen that the functors $E^{i}(-)$ play an essential role in the dimension theory of $\Lambda$-modules. In this section we will mention some techniques which sometimes allow to calculate these adjoints in applications when $\Lambda$ is the Iwasawa algebra $\Lambda(G)$ of a profinite group $G$. A part of the results stems from U. Jannsen ([22]) whom I would like to thank heartily for giving me his manuscript on a spectral sequence for Iwasawa adjoints ([23]).

We shall write $\mathcal{D}(G)$ and $\mathcal{C}(G)$ for the categories of discrete and compact $\Lambda$ modules, respectively, whereas we denote the full subcategories of cofinitely and finitely generated modules by $\mathcal{D}_{c f g}(G)$ and $\mathcal{C}_{f g}(G)$, respectively.

Now, let $G=H \times \Gamma$ be the product of profinite groups $H$ and $\Gamma$. Assume that $\Lambda(G)$ is Noetherian and that $\Gamma$ is separable, i.e. it possesses a countable ordered system of open normal subgroups $\Gamma_{n}$ as a basis of open neighborhoods of $1 \epsilon \Gamma$. Let $\left(\mathcal{D}_{c f g}(G)\right)^{\mathbb{N}}$ be the category of inverse systems in $\mathcal{D}_{c f g}(G)$ and consider the left exact functor

$$
T_{\Gamma}: \mathcal{D}_{c f g}(G) \rightarrow\left(\mathcal{D}_{c f g}(G)\right)^{\mathbb{N}}
$$

which sends $B$ to the inverse system $\left\{B^{\Gamma_{n+1}} \stackrel{N_{\Gamma_{n} / \Gamma_{n+1}}}{\longrightarrow} B^{\Gamma_{n}}\right\}$, and

$$
{\underset{\lim }{n}}_{n} \operatorname{Hom}_{\Lambda(H)}\left(-^{\vee}, \Lambda(H)\right):\left(\mathcal{D}_{c f g}(G)\right)^{\mathbb{N}} \rightarrow \Lambda(G) \text {-Mod. }
$$

Here the action of $\Gamma$ on $f \in \operatorname{Hom}_{\Lambda(H)}(M, \Lambda(H))$ for $M \in \mathcal{C}(G)$ is defined by $(\gamma f)(m):=f\left(\gamma^{-1} m\right)$, whereas $h \epsilon H$ acts by the rule $(h f)(M):=f(m) h^{-1}$ as usual.

Since the category $\left(\mathcal{D}_{c f g}(G)\right)^{\mathbb{N}}$ has enough injectives, because $\mathcal{D}_{c f g}(G)$ has ([21], Proposition 1.1), we can form the right derived functors

$$
R^{i} T_{\Gamma}(B)=\left\{\mathrm{H}^{i}\left(\Gamma_{n+1}, B\right) \stackrel{c o r}{\longrightarrow} \mathrm{H}^{i}\left(\Gamma_{n}, B\right)\right\}
$$

and

$$
R^{i}\left(\overleftarrow{\lim }_{n} \operatorname{Hom}_{\Lambda(H)}\left(B^{\vee}, \Lambda(H)\right)\right),
$$


which equals

$$
\overleftarrow{\lim }_{n} R^{i} \operatorname{Hom}_{\Lambda(H)}\left(B^{\vee}, \Lambda(H)\right)
$$

(cf. [21] Proposition 1.2, 1.3), if we restrict ourselves to elements of the subcategory $\left(\mathcal{D}^{\prime}\right)^{\mathbb{N}}$ where $\mathcal{D}^{\prime}$ is the abelian subcategory of $\mathcal{D}_{c f g}(G)$ consisting of $\Lambda(G)$-modules, which are cofinitely generated over $\Lambda(H)$. Indeed, in this case, the modules $\operatorname{Hom}_{\Lambda(H)}\left(B_{n}^{\vee}, \Lambda(H)\right)$ are compact, i.e. the inverse limit functor is exact on the corresponding inverse systems. Since $R^{i} \operatorname{Hom}_{\Lambda(H)}(-, \Lambda(H))$ extends the functors $\mathrm{E}_{\Lambda(H)}^{i}(-)$ naturally from $\mathcal{C}(H)$ to $\mathcal{C}_{f g}(G)$, we will write also $\mathrm{E}_{\Lambda(H)}^{i}(-)$ for the first functor. Note that it is endowed with a natural $\Gamma$-action.

Lemma 4.1. The functor $T_{\Gamma}$ sends injectives to ${\underset{n}{\lim _{n}}}_{\operatorname{Hom}_{\Lambda(H)}}\left(-^{\vee}, \Lambda(H)\right)$-acylics.

Proof. It suffices to prove that $\mathbb{Z}_{p} \llbracket H \rrbracket\left[\Gamma / \Gamma_{n}\right]$ is $\operatorname{Hom}_{\Lambda(H)}(-, \Lambda(H))$-acyclic. But, for any resolution of $\mathbb{Z}_{p} \llbracket H \rrbracket\left[\Gamma / \Gamma_{n}\right]$ by $\Lambda(G)$-projectives

$$
P^{\bullet} \rightarrow \mathbb{Z}_{p} \llbracket H \rrbracket\left[\Gamma / \Gamma_{n}\right],
$$

the sequence

$$
0 \rightarrow \operatorname{Hom}_{\Lambda(H)}\left(\mathbb{Z}_{p} \llbracket H \rrbracket\left[\Gamma / \Gamma_{n}\right], \Lambda(H)\right) \rightarrow \operatorname{Hom}_{\Lambda(H)}\left(P^{\bullet}, \Lambda(H)\right)
$$

is exact, because both, $\mathbb{Z}_{p} \llbracket H \rrbracket\left[\Gamma / \Gamma_{n}\right]$ and the $P^{i}$, are projectives in $\mathcal{C}(H)$ (cf. [27] (5.3.13)). The result follows by taking homology.

The Grothendieck spectral sequence for the composition of the above functors gives

Theorem 4.2. With notation as above, there is a convergent cohomological spectral sequence

$$
\overleftarrow{\lim }_{n} \mathrm{E}_{\Lambda(H)}^{i}\left(\mathrm{H}^{j}\left(\Gamma_{n}, B\right)^{\vee}\right) \Rightarrow \mathrm{E}_{\Lambda(G)}^{i+j}\left(B^{\vee}\right)
$$

for any $B$ in $\mathcal{D}_{c f g}(G)$.

Note that all modules that occur in the spectral sequence are compact $\Lambda(G)$ modules.

Proof. The functor $\mathrm{E}_{\Lambda(G)}^{0}(-)$ is the composition of the functors $T_{\Gamma}$ and $\underset{\leftarrow}{\lim } \operatorname{Hom}_{\Lambda(H)}\left(-^{\vee}, \Lambda(H)\right)$, because by Lemma 4.3 we have isomorphisms of $\Lambda(G)$-modules

$$
\begin{aligned}
\mathrm{E}_{\Lambda(G)}^{0}\left(B^{\vee}\right) & \left.=\operatorname{Hom}_{\Lambda(G)}\left(B^{\vee}, \mathbb{Z}_{p} \llbracket H \rrbracket\right) \llbracket \Gamma \rrbracket\right) \\
& =\overleftarrow{n}_{n}^{\lim } \operatorname{Hom}_{\mathbb{Z}_{p} \llbracket H \rrbracket\left[\Gamma / \Gamma_{n}\right]}\left(\left(B^{\vee}\right)_{\Gamma_{n}}, \mathbb{Z}_{p} \llbracket H \rrbracket\left[\Gamma / \Gamma_{n}\right]\right) \\
& =\overleftarrow{\lim }_{n}^{\lim } \operatorname{Hom}_{\Lambda(H)}\left(\left(B^{\Gamma_{n}}\right)^{\vee}, \Lambda(H)\right) .
\end{aligned}
$$

Now the result follows by Lemma 4.1. 
Recall that there is a canonical $\Lambda(H)$-homomorphism

$$
\pi_{n}: \mathbb{Z}_{p} \llbracket H \rrbracket\left[\Gamma / \Gamma_{n}\right] \rightarrow \mathbb{Z}_{p} \llbracket H \rrbracket, \sum_{g \in \Gamma / \Gamma_{n}} a_{g} g \Gamma_{n} \mapsto a_{1},
$$

and, for any $m \geq n$, a canonical $\Lambda(G)$-homomorphism $p_{m, n}: \mathbb{Z}_{p} \llbracket H \rrbracket\left[\Gamma / \Gamma_{m}\right] \rightarrow$ $\mathbb{Z}_{p} \llbracket H \rrbracket\left[\Gamma / \Gamma_{n}\right]$ which sums up the coefficients of the same $\Gamma_{n}$-cosets.

Lemma 4.3. The homomorphisms $\pi_{n}$ and $p_{m, n}$ induce a commutative diagram of $\Lambda(G)$-modules:

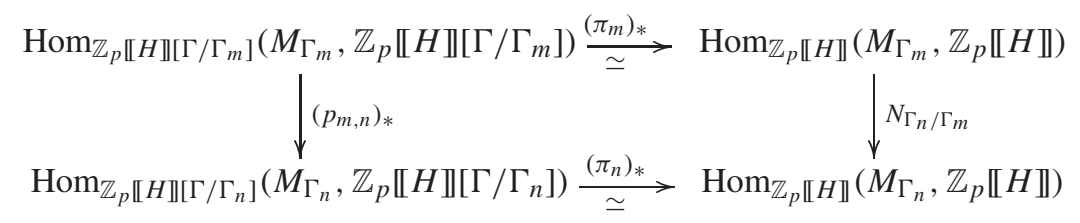

Proof. It is easily checked that the diagram commutes and that the inverse of $\left(\pi_{n}\right)_{*}$ is given by $\psi \mapsto\left(m \mapsto \sum_{g \in \Gamma / \Gamma_{n}} \psi\left(g^{-1} m\right) g \Gamma_{n}\right)$. (Note that the $\Gamma$ invariance of a homomorphism $\phi(m)=\sum \phi(m)_{g} g \Gamma_{n}$ is equivalent to $\phi\left(\gamma^{-1} m\right)_{1}=$ $\phi(m)_{\gamma}$ for all $\gamma \in \Gamma$.) Recalling that $\gamma \in \Gamma$ acts by $(\gamma \phi)(m):=\phi\left(\gamma^{-1} m\right)$ on $\operatorname{Hom}_{\mathbb{Z}_{p} \llbracket H \rrbracket\left[\Gamma / \Gamma_{n}\right]}\left(M_{\Gamma_{n}}, \mathbb{Z}_{p} \llbracket H \rrbracket\left[\Gamma / \Gamma_{n}\right]\right)$, it is also immediate that $\left(\pi_{n}\right)_{*}$ is $\Lambda(G)$ invariant.

Corollary 4.4. If $\Gamma$ contains an open subgroup of index prime to $p$ and isomorphic to $\mathbb{Z}_{p}$, then there is a long exact sequence of $\Lambda(G)$-modules

${\underset{\lim }{n}}_{n}^{i} \mathrm{E}_{\Lambda(H)}^{i}\left(M_{\Gamma_{n}}\right) \rightarrow \mathrm{E}_{\Lambda(G)}^{i}(M) \rightarrow \underset{\leftarrow}{\lim } \mathrm{E}_{\Lambda(H)}^{i-1}\left(M^{\Gamma_{n}}\right) \rightarrow \underset{n}{\lim } \mathrm{E}_{\Lambda(H)}^{i+1}\left(M_{\Gamma_{n}}\right) \rightarrow \mathrm{E}_{\Lambda(G)}^{i+1}(M)$

Now we are going to present further spectral sequences due to U. Jannsen which were in some sense the models for the first one proved in this section. The next one describes the Iwasawa adjoints of certain cohomology groups associated with $p$-adic representations. So let $G$ be a profinite group and $G_{\infty}$ a closed subgroup, such that its quotient has a countable basis of neighbourhoods of identity, i.e. there is a countable family $G_{n}, G_{\infty} \subseteq G_{n} \subseteq G$, with $\bigcap_{n} G_{n}=G_{\infty}$. Furthermore, let $A=\left(\mathbb{Q}_{p} / \mathbb{Z}_{p}\right)^{r}$ for some $r \geq 1$ with some continuous action of $G$. We shall write

$$
T_{p} A=\operatorname{Hom}\left(\mathbb{Q}_{p} / \mathbb{Z}_{p}, A\right) \cong \overleftarrow{m}_{\lim _{m}} p^{m} A
$$

for the Tate module of $A$. Then there is the following convergent spectral sequence ([23]):

\section{Theorem 4.5 (Jannsen).}

$$
E_{2}^{p, q}=\mathrm{E}^{p}\left(\mathrm{H}^{q}\left(G_{\infty}, A\right)^{\vee}\right) \Rightarrow \underset{n}{\lim } \mathrm{H}^{p+q}\left(G_{n}, T_{p} A\right)
$$


Corollary 4.6. Assume $\operatorname{cd}_{p}(G) \leq 2$. Then the exact sequence of low degrees degenerates to

$$
\begin{aligned}
& 0 \longrightarrow \mathrm{E}^{1}\left(A\left(k_{\infty}\right)^{\vee}\right) \longrightarrow \overleftarrow{n}_{n}^{\lim } \mathrm{H}^{1}\left(G_{n}, T_{p} A\right) \longrightarrow \mathrm{E}^{0}\left(\mathrm{H}^{1}\left(G_{\infty}, A\right)^{\vee}\right) \longrightarrow \\
& \mathrm{E}^{2}\left(A\left(k_{\infty}\right)^{\vee}\right) \longrightarrow \operatorname{ker}\left(\overleftarrow{n}_{n}^{\lim } \mathrm{H}^{2}\left(G_{n}, T_{p} A\right) \longrightarrow \mathrm{E}^{0}\left(\mathrm{H}^{2}\left(G_{\infty}, A\right)^{\vee}\right)\right) \longrightarrow \\
& \mathrm{E}^{1}\left(\mathrm{H}^{1}\left(G_{\infty}, A\right)^{\vee}\right) \longrightarrow \mathrm{E}^{3}\left(A\left(k_{\infty}\right)^{\vee}\right) \longrightarrow 0 .
\end{aligned}
$$

The next result, which relates the (compact) $\Lambda$-modules $\mathrm{E}^{i}(M)$ to the discrete $G$-modules

$$
D_{i}\left(M^{\vee}\right):=\underset{U \subseteq_{o} G}{\lim _{\longrightarrow}} H^{i}\left(U, M^{\vee}\right)^{*}, i \geq 0,
$$

is derived by some spectral sequences, too, but we only state the associated long, respectively short, exact sequences:

Theorem 4.7 (Jannsen). Let $G$ be a profinite group such that $\Lambda(G)$ is Noetherian. Then, for any finitely generated $\Lambda$-module $M$, there are functorial exact sequences

(i)

$$
0 \longrightarrow D_{i}\left(M^{\vee}\right) \otimes_{\mathbb{Z}_{p}} \mathbb{Q}_{p} / \mathbb{Z}_{p} \longrightarrow \mathrm{E}^{i}(M)^{\vee} \longrightarrow \operatorname{tor}_{\mathbb{Z}_{p}} D_{i-1}\left(M^{\vee}\right) \longrightarrow 0,
$$

(ii)

for all $i$, where by definition $D_{i}\left(M^{\vee}\right)=0$ for $i<0$.

$$
\longrightarrow \mathrm{E}^{i}(M)^{\vee} \longrightarrow \underset{m}{\lim } D_{i}\left(p^{m}\left(M^{\vee}\right)\right) \longrightarrow \underset{m}{\lim } D_{i-2}\left(M^{\vee} / p^{m}\right) \longrightarrow \mathrm{E}^{i-1}(M)^{\vee} \longrightarrow,
$$

and the following isomorphisms

(iii) $\mathrm{E}^{i}\left(M / \text { tor }_{\mathbb{Z}_{p}} M\right)^{\vee} \cong \underset{m}{\lim } D_{i}\left(p^{m}\left(M^{\vee}\right)\right)$,

(iv) $\mathrm{E}^{i}\left(\operatorname{tor}_{\mathbb{Z}_{p}} M\right)^{\vee} \cong \underset{m}{\lim } D_{i-1}\left(M^{\vee} / p^{m}\right)$.

Proof. See [22] 2.1, 2.2 or [27] Theorem 5.4.12.

For the duality theorem in the next section the following corollary will be crucial.

Corollary 4.8. Assume that $G$ is a duality group at $p$ of dimension $n$ with dualizing module $D_{n}^{(p)}=\underset{m}{\lim } D_{n}\left(\mathbf{Z} / p^{m} \mathbf{Z}\right)$. Then the following holds:

(i) If $M$ is $\Lambda$-module which is free of finite rank as $\mathbb{Z}_{p}$-module, then

$$
\mathrm{E}^{i}(M)^{\vee} \cong\left\{\begin{array}{cl}
\underset{m}{\lim } D_{n}\left(\left(M / p^{m}\right)^{\vee}\right) \cong M \otimes_{\mathbb{Z}_{p}} D_{n}^{(p)} & \text { if } i=n, \\
0 & \text { otherwise. }
\end{array}\right.
$$


(ii) If $N$ is a finite p-primary $\Lambda$-module, then

$$
\mathrm{E}^{i}(N)^{\vee} \cong\left\{\begin{array}{cl}
\operatorname{Hom}_{\mathbb{Z}_{p}}\left(N^{\vee}, D_{n}^{(p)}\right) & \text { if } i=n+1, \\
0 & \text { otherwise. }
\end{array}\right.
$$

Proof. See [22] 2.6 or [27] 5.4.14.

Proposition 4.9. Let $G$ be a compact p-adic analytic group without p-torsion, $H \subseteq G$ a closed subgroup and $M$ a finitely generated $\Lambda(H)$-module. If $d_{\Lambda(G)}$ (resp. $d_{\Lambda(H)}$ ) denotes the (projective or $\delta$-) dimension of $\Lambda(G)($ resp. $\Lambda(H))$, then the following holds:

(i) $j_{\Lambda(G)}\left(\operatorname{Ind}_{G}^{H} M\right)=j_{\Lambda(H)}(M)$,

(ii) $\delta_{\Lambda(G)}\left(\operatorname{Ind}_{G}^{H} M\right)=\delta_{\Lambda(H)}(M)+d_{\Lambda(G)}-d_{\Lambda(H)}$,

(iii) $\operatorname{pd}_{\Lambda(G)}\left(\operatorname{Ind}_{G}^{H} M\right)=\operatorname{pd}_{\Lambda(H)}(M)$.

Proof. This is a consequence of 2.7, 3.5, (ii), and 6.3.

Lemma 4.10. Assume that $G=H \times \Gamma$ is a p-adic Lie group without p-torsion where $\Gamma$ contains an open subgroup of index prime to $p$ which is isomorphic to $\mathbb{Z}_{p}$. Let $M \in \mathcal{C}(G)$ be finitely generated and torsion as $\Lambda(H)$-module. Then $M$ is a pseudo-null $\Lambda(G)$-module.

Proof. By the Corollary 4.4, there is an exact sequence

$$
0 \longrightarrow \overleftarrow{\lim }_{n} \mathrm{E}_{\Lambda(H)}^{1}\left(M_{\Gamma_{n}}\right) \longrightarrow \mathrm{E}_{\Lambda(G)}^{1}(M) \longrightarrow \underset{n}{\lim } \mathrm{E}_{\Lambda(H)}^{0}\left(M^{\Gamma_{n}}\right)=0
$$

So, if we can show that the left term vanishes, we are done, because then $\mathrm{E}^{1} \mathrm{E}^{1}(M)=$ $0=\mathrm{E}^{0} \mathrm{E}^{0}(M)$. Consider the commutative exact diagram

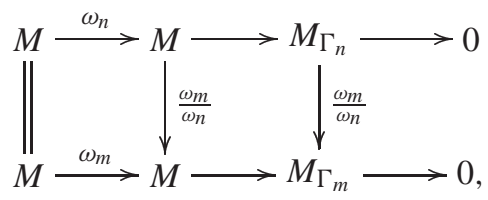

where $\omega_{n}=\gamma^{p^{n}}-1$ for some generator $\gamma$ of $\mathbb{Z}_{p} \subseteq \Gamma$. Since $M$ is assumed to be $\Lambda(H)$-torsion, we get the commutative exact diagram

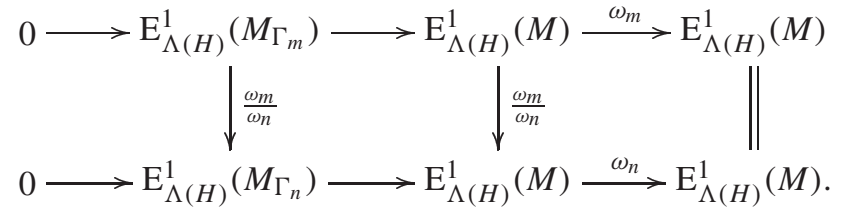

Passing to the limit, we obtain

$$
\overleftarrow{\lim }_{n} \mathrm{E}_{\Lambda(H)}^{1}\left(M_{\Gamma_{n}}\right) \subseteq \underset{\longleftarrow_{n}}{\lim } \mathrm{E}_{\Lambda(H)}^{1}(M)={\underset{\leftarrow}{n}}_{m \geq n} \bigcap_{m \geq n} \frac{\omega_{m}}{\omega_{n}} \mathrm{E}_{\Lambda(H)}^{1}(M)=0,
$$

because $\frac{\omega_{m}}{\omega_{n}}$ tends to zero. 
Remark 4.11. The same arguments show that $\delta_{G}(M) \leq \delta_{H}(M)$ for any finitely generated $\Lambda(H)$-module $M$.

Besides the case $G=\mathbb{Z}_{p}^{d}$ these results apply also to the following situation where $G$ is an open subgroup of $G l_{d}\left(\mathbb{Z}_{p}\right), d$ is prime to $p$, such that the determinant takes values in $\Gamma:=\operatorname{det}(G) \subseteq \mathbb{Z}_{p} \subseteq \mathbb{Z}_{p}^{*}$. at least if . Indeed, we have the following exact commutative diagram

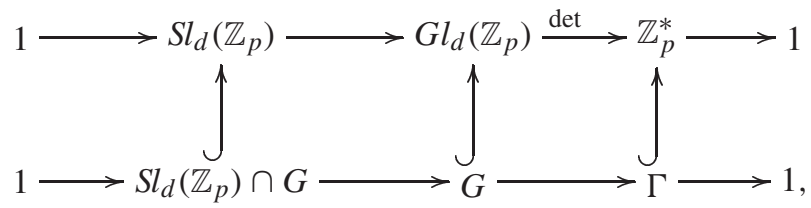

in which the lower sequence possesses the following splitting

$$
s: \Gamma \cong \mathbb{Z}_{p} \rightarrow G, a \mapsto\left(\begin{array}{llll}
a^{1 / d} & & & \\
& a^{1 / d} & & \\
& & \ddots & \\
& & & a^{1 / d}
\end{array}\right)
$$

(Note that $\Gamma \cong \mathbb{Z}_{p}$ is considered as subgroup of the units and that $\mathbb{Z}_{p}$ is uniquely $d$-divisible. Furthermore, if the image of this homomorphism is not contained in $G$, we just apply the theory to an open subgroup $U$ of $G$ which fulfills this condition with respect to $\operatorname{det}(U)$ and contains $H:=S l_{d}\left(\mathbb{Z}_{p}\right) \cap G$. Such $U$ always exists because $G l_{d}\left(\mathbb{Z}_{p}\right)$ is $p$-adic analytic, i.e. the lower $p$-series forms a basic of neighborhoods of the neutral element. Hence at least for some $m$ the image of $p^{m} \Gamma$ is contained in $G: s\left(a^{p^{m}}\right)=s(a)^{p^{m}} \in P_{m}\left(G l_{d}\right) \subseteq G$. Take $U:=\operatorname{det}^{-1}\left(p^{m} \Gamma\right) \cap G$.) Since the splitting takes values in the center of $G$, we get a presentation of $G$ as the direct product $G=H \times \mathbb{Z}_{p}$.

\section{Local duality}

In this and the following section let $\Lambda=\Lambda(G)=\mathbb{Z}_{p} \llbracket G \rrbracket$ be the completed group algebra over $\mathbb{Z}_{p}$, where $G$ is a pro- $p$ Poincaré group, such that $\Lambda$ is Noetherian, $\mathfrak{M}$ the maximal ideal of $\Lambda$ and $k=\Lambda / \mathfrak{M} \cong \mathbb{F}_{p}$ its finite residue class field. It is well known that the global homological dimension of $\Lambda$ is $d=\operatorname{cd}(G)+1$. By $\Lambda$-Mod we denote the category of (abstract) modules over the (abstract) ring $\Lambda$ and we write $\Lambda$-mod for the full subcategory of finitely generated modules. In the sequel we will use frequently the equivalence of the latter category with the category of finitely generated compact modules.

Definition 5.1. For a finitely generated $\Lambda$-module $M$, we define the depth by

$$
\operatorname{depth}(M):=\min \left\{i \mid \operatorname{Ext}_{\Lambda}^{i}(k, M) \neq 0\right\}
$$


Recall that for a commutative Noetherian ring $\Lambda$ the $I$-depth $\operatorname{depth}_{I}(M)$ of a finitely generated $\Lambda$-module $M$ with respect to an ideal $I$ is the maximal length of a $M$-regular sequence in $I$. For a local ring the $\operatorname{depth}(M)$ is $\operatorname{depth}_{\mathfrak{M}}(M)$, while the grade defined in 3.3 is $j(M)=\operatorname{depth}_{\operatorname{ann}(M)}(\Lambda)$, where $\operatorname{ann}(M)$ is the annihilator of $M$ in $\Lambda$.

We consider the additive functor $\Gamma_{\mathfrak{M}}(-): \Lambda$-Mod $\rightarrow \Lambda$-Mod defined by $\Gamma_{\mathfrak{M}}(M):=\left\{x \in M \mid \mathfrak{M}^{l} x=0\right.$ for some 1$\}$ and state some basic properties:

Lemma 5.2. (i) $\Gamma_{\mathfrak{M}}(M)=\underset{l}{\lim } \operatorname{Hom}_{\Lambda}\left(\Lambda / \mathfrak{M}^{l}, M\right)$, in particular, the functor $\Gamma_{\mathfrak{M}}(-)$ is left exact.

(ii) The restriction of $\Gamma_{\mathfrak{M}}$ to $\Lambda$-mod equals $\mathrm{T}_{0}$, i.e. $\Gamma_{\mathfrak{M}}(M)$ is the maximal finite submodule of $M$, if the latter module is finitely generated.

Proof. Since $\operatorname{Hom}_{\Lambda}\left(\Lambda / \mathfrak{M}^{l}, M\right)=\left\{x \in M \mid \mathfrak{M}^{l} x=0\right\}$, the first statement is obvious. If $M$ is finitely generated, there is some $l$ such that $\mathfrak{M}^{l} \mathrm{~T}_{0}(M)=0$, i.e. $\mathrm{T}_{0}(M) \subseteq \Gamma_{\mathfrak{M}}(M)$. On the other hand $\Lambda / \mathfrak{M}^{l}$ is a finite ring. Therefore $\Lambda x \subseteq$ $\mathrm{T}_{0}(M)$ holds for any $x \in \Gamma_{\mathfrak{M}}(M)$.

Since $\Lambda$-Mod has sufficiently many injectives, we can form the right derived functors

$$
\mathrm{H}_{\mathfrak{M}}^{i}(-)=R^{i} \Gamma_{\mathfrak{M}}(-)=\underset{l}{\lim } \operatorname{Ext}_{\Lambda}^{i}\left(\Lambda / \mathfrak{M}^{l}, M\right)
$$

(noting the exactness of direct limits in $\Lambda$-Mod). We write

$$
\Lambda-\operatorname{Mod}_{\mathfrak{M}}, \Lambda-\bmod _{\mathfrak{M}}
$$

for the full subcategory of $\Lambda$-Mod, $\Lambda$-mod respectively, consisting of those modules $M$, for which $\mathrm{H}_{\mathfrak{M}}^{0}(M)=M$ holds.

$$
\mathcal{D}(\Lambda \text {-Mod })(\text { resp. } \mathcal{C}(\Lambda-\operatorname{Mod}))
$$

means the category of discrete (resp. compact) $\Lambda$-modules, where $\Lambda$ is endowed with its canonical $(\mathfrak{m}, I)$-topology.

Lemma 5.3. $\mathrm{H}_{\mathfrak{M}}^{i}(-)$ commutes with direct limits.

Proof. Choose a resolution $P_{\bullet}$ of $\Lambda / \mathfrak{M}^{l}$ by finitely generated projectives in order to calculate $\operatorname{Ext}_{\Lambda}^{i}\left(\Lambda / \mathfrak{M}^{l}, M\right)$. Since $\operatorname{Hom}_{\Lambda}\left(P_{j},-\right)$ commutes with direct limits (as $P_{j}$ is finitely generated, i.e. any homomorphism $\phi: P_{j} \rightarrow \underset{i}{\lim } M_{i}$ factors over some $\left.M_{i}\right), \operatorname{Ext}_{\Lambda}^{i}\left(\Lambda / \mathfrak{M}^{l},-\right)$ does also and the lemma follows.

Proposition 5.4. The forgetful functor defines an equivalence of categories

$$
\mathcal{D}(\Lambda-\operatorname{Mod}) \cong \Lambda-\operatorname{Mod}_{\mathfrak{M}}
$$

Proof. Both categories consists exactly of direct limits of finite modules (cf. [27, Proposition (5.2.4)] for $\mathcal{D}(\Lambda-$ Mod $)$ ). 
Lemma 5.5. (i) $\mathrm{H}_{\mathfrak{M}}^{i}(\Lambda-M o d) \subseteq \Lambda-M o d_{\mathfrak{M}} \cong \mathcal{D}(\Lambda-M o d)$ for all $i \geq 0$.

(ii) For any $M \in \Lambda$-mod, it holds $\operatorname{depth}(M)=\min \left\{i \mid \mathrm{H}_{\mathfrak{M}}^{i}(M) \neq 0\right\}$.

(iii) $\operatorname{depth}(\Lambda)=d$ and $\mathrm{H}_{\mathfrak{M}}^{d}(\Lambda)=\Lambda^{\vee}$.

(iv) $\operatorname{Hom}_{\Lambda}\left(M, \mathrm{H}_{\mathfrak{M}}^{d}(\Lambda)\right) \cong M^{\vee}$ for all $M$ in $\Lambda-M_{\mathfrak{M}}$ or in $\Lambda$-mod, in particular, $\mathrm{H}_{\mathfrak{M}}^{d}(\Lambda)$ is an injective $\Lambda$-module.

Proof. Since $\mathrm{H}_{\mathfrak{M}}^{i}(-)$ are the derived functors of $\mathrm{H}_{\mathfrak{M}}^{0}(-)$, it suffices to prove (i) for the latter functor. But in this case the statement holds just by definition.

Now we will prove (ii) and set $k=\min \left\{i \mid \mathrm{H}_{\mathfrak{M}}^{i}(M) \neq 0\right\}$. Since $\operatorname{Ext}_{\Lambda}^{i}\left(\Lambda / \mathfrak{M}^{l}, M\right)=0$ for all $i<\operatorname{depth}(M)$ (note that $\Lambda / \mathfrak{M}^{l}$ has a finite composition series with subquotients isomorphic to $k$ ), it holds $\operatorname{depth}(M) \leq k$. So we only have to prove that $\mathrm{H}_{\mathfrak{M}}^{j}(M) \neq 0$ for $j=\operatorname{depth}(M)<\infty$. But the short exact sequences

$$
0 \longrightarrow \mathfrak{M} / \mathfrak{M}^{l} \longrightarrow \Lambda / \mathfrak{M}^{l} \longrightarrow k \longrightarrow 0
$$

induce the long exact sequences

$$
0=\operatorname{Ext}_{\Lambda}^{j-1}\left(\mathfrak{M} / \mathfrak{M}^{l}, M\right) \longrightarrow \operatorname{Ext}_{\Lambda}^{j}(k, M) \longrightarrow \operatorname{Ext}_{\Lambda}^{j}\left(\Lambda / \mathfrak{M}^{l}, M\right) \longrightarrow \cdots,
$$

i.e. $0 \neq \operatorname{Ext}_{\Lambda}^{j}(k, M) \subseteq \mathrm{H}_{\mathfrak{M}}^{j}(M)$.

Using 4.8 and denoting the character of the dualizing module by $\chi$, we calculate

$$
\mathrm{H}_{\mathfrak{M}}^{i}(\Lambda)=\underset{l}{\lim } \mathrm{E}^{i}\left(\Lambda / \mathfrak{M}^{l}\right)=\left\{\begin{array}{cc}
\underset{l}{\lim }\left(\Lambda / \mathfrak{M}^{l}(\chi)\right)^{\vee}=\Lambda^{\vee} & \text { if } i=d \\
0 & \text { otherwise, }
\end{array}\right.
$$

whence (iii) follows. In order to prove (iv) first let $M$ be in $\Lambda$-Mod $\mathfrak{M}$, i.e. $M=$ $\underset{\lim }{\longrightarrow} M_{i}$ for some finite $\Lambda$-modules $M_{i}$. Then, noting that $M_{i}$ is a $\Lambda / \mathfrak{M}^{l(i)}$-module $\vec{i}$

for some $l(i)$ and using the adjunction of "Hom and $\otimes$ ",

$$
\begin{aligned}
\operatorname{Hom}_{\Lambda}\left(M, \mathrm{H}_{\mathfrak{M}}^{d}(\Lambda)\right) & =\operatorname{Hom}_{\Lambda}\left(\underset{i}{\lim } M_{i}, \underset{l}{\lim }\left(\Lambda / \mathfrak{M}^{l}\right)^{\vee}\right) \\
& =\overleftarrow{l i m}_{i}^{\lim } \operatorname{Hom}_{\Lambda}\left(M_{i}, \underset{l}{\lim }\left(\Lambda / \mathfrak{M}^{l}\right)^{\vee}\right) \\
& =\overleftarrow{l}_{i}^{\lim } \operatorname{Hom}_{\Lambda}\left(M_{i}, \operatorname{Hom}_{\mathbb{Z}_{p}}\left(\Lambda / \mathfrak{M}^{l(i)}, \mathbb{Q}_{p} / \mathbb{Z}_{p}\right)\right) \\
& =\overleftarrow{l i m}_{i}^{\lim } \operatorname{Hom}_{\mathbb{Z}_{p}}\left(M_{i}, \mathbb{Q}_{p} / \mathbb{Z}_{p}\right) \\
& =M^{\vee} .
\end{aligned}
$$


Now let $M$ be in $\Lambda$-mod . Then, noting that $\operatorname{Hom}_{\Lambda}(M,-)$ commutes with direct limits, because $M$ is finitely generated,

$$
\begin{aligned}
& \operatorname{Hom}_{\Lambda}\left(M, \mathrm{H}_{\mathfrak{M}}^{d}(\Lambda)\right)=\operatorname{Hom}_{\Lambda}\left(M, \underset{l}{\lim }\left(\Lambda / \mathfrak{M}^{l}\right)^{\vee}\right) \\
& =\underset{l}{\lim } \operatorname{Hom}_{\Lambda}\left(M,\left(\Lambda / \mathfrak{M}^{l}\right)^{\vee}\right) \\
& =\underset{l}{\lim } \operatorname{Hom}_{\Lambda}\left(M / \mathfrak{M}^{l}, \operatorname{Hom}_{\mathbb{Z}_{p}}\left(\Lambda / \mathfrak{M}^{l}, \mathbb{Q}_{p} / \mathbb{Z}_{p}\right)\right) \\
& =\underset{l}{\lim } \operatorname{Hom}_{\mathbb{Z}_{p}}\left(M / \mathfrak{M}^{l}, \mathbb{Q}_{p} / \mathbb{Z}_{p}\right) \\
& =M^{\vee} \text {. }
\end{aligned}
$$

After this technical preparations we are able to prove the following

Theorem 5.6. Let $G$ be a pro-p Poincaré group with $d:=\operatorname{cd}(G)+1<\infty$ and such that $\Lambda=\Lambda(G)$ is Noetherian. Then, for any $M \in \Lambda$-mod,

$$
\mathrm{E}^{i}(M) \cong \operatorname{Hom}_{\Lambda}\left(\mathrm{H}_{\mathfrak{M}}^{d-i}(M), \mathrm{H}_{\mathfrak{M}}^{d}(\Lambda)\right) \cong \mathrm{H}_{\mathfrak{M}}^{d-i}(M)^{\vee}=: T^{i}(M)
$$

Proof. Consider the right exact contravariant additive functor $T^{0}(-)=\mathrm{H}_{\mathfrak{M}}^{d}(M)^{\vee}$ on $\Lambda$-mod (note that $\mathrm{H}_{\mathfrak{M}}^{i}(M)=0$ for all $i>d$ as $\Lambda$ has global dimension $d$ ). By [30, Theorem 3.36 and Remarks] there is a natural equivalence of functors

$$
T^{0}(-) \cong \operatorname{Hom}_{\Lambda}\left(-, T^{0}(\Lambda)\right) \cong \operatorname{Hom}_{\Lambda}(-, \Lambda)
$$

on $\Lambda$-mod. Therefore, it suffices to show that the functors $T^{i}(-)$ are the left derived functors of $T^{0}(-)$. But $\left\{T^{i}(-)\right\}_{i>0}$ is a universal $\delta$-functor because they are effaceable by projectives in $\Lambda$-mod (Since $T^{0}$ is additive, it is sufficient to verify that $\mathrm{H}_{\mathfrak{M}}^{i}(\Lambda)=0$ for all $i<d$, which is done by Lemma 5.5 (iii)).

\section{Auslander-Buchsbaum equality}

In this section we assume the same conditions on $\Lambda$ as in the previous one and, under this conditions, we are going to prove the Auslander-Buchsbaum equality

$$
\operatorname{pd}(M)+\operatorname{depth}(M)=\operatorname{depth}(\Lambda)
$$

for all $M \epsilon \Lambda$-mod. In the theory of commutative local rings this equality can be proved using regular sequences. Since this concept is lacking in the noncommutative theory, we will have to replace it by homological methods, i.e. we will work in derived categories. Our proof is analogous to Jørgensen's proof of the Auslander-Buchsbaum equality in the case of (non-commutative) graded algebras over a field (cf. [24]). 
First, we recall the definitions of total Hom and total tensor product. Let $X, Y \in \mathbf{K}(\Lambda$-Mod $)$ and define

$$
\left(\operatorname{Hom}_{\Lambda}(X, Y)\right)^{n}=\prod_{i \in \mathbb{Z}} \operatorname{Hom}_{\Lambda}\left(X^{i}, Y^{i+n}\right), d^{n}=\prod_{i}\left(d_{X}^{i-1}+(-1)^{n+1} d_{Y}^{i+n}\right)
$$

and

$$
\left(X \otimes_{\Lambda} Y\right)^{n}=\bigoplus_{i+j=n} X^{i} \otimes_{\Lambda} Y^{j}, d^{n}=\bigoplus_{i+j=n}\left(d_{X}^{i} \otimes 1+(-1)^{n} \otimes d_{Y}^{j}\right) .
$$

They become bifunctors

$$
\begin{gathered}
\operatorname{Hom}_{\Lambda}(-,-): \mathbf{K}(\Lambda-\operatorname{Mod})^{o p} \times \mathbf{K}(\Lambda-\operatorname{Mod}) \rightarrow \mathbf{K}\left(\mathbb{Z}_{p}-\text { Mod }\right), \\
-\otimes_{\Lambda}-: \mathbf{K}(\operatorname{Mod}-\Lambda) \times \mathbf{K}(\Lambda-\operatorname{Mod}) \rightarrow \mathbf{K}\left(\mathbb{Z}_{p}-\text { Mod }\right),
\end{gathered}
$$

where we denote by Mod- $\Lambda$ the category of right $\Lambda$-modules. Note that the latter category is equivalent to $\Lambda$-Mod due to the involution on the group algebra $\Lambda$. Moreover, if $Y$ is a complex of bi-modules, then the values of $\operatorname{Hom}_{\Lambda}(-, Y)$ are in $\mathbf{K}(\operatorname{Mod}-\Lambda)$, if $X$ is a complex of bi-modules, then $X \otimes_{\Lambda}-$ has values in $\mathbf{K}(\Lambda$-Mod $)$.

Since $\Lambda$-Mod has enough projectives, the derived functors exist (cf. [17, Chap. I, Theorem 5.1] or [36, Theorem 10.5.6]):

$$
\mathbf{R H o m}_{\Lambda}(-,-): \mathbf{D}^{-}(\Lambda-\operatorname{Mod})^{o p} \times \mathbf{D}(\Lambda-\operatorname{Mod}) \rightarrow \mathbf{D}\left(\mathbb{Z}_{p}-\operatorname{Mod}\right),
$$

respectively

$$
\mathbf{R H o m}_{\Lambda}(-,-): \mathbf{D}^{-}(\Lambda-\operatorname{Mod})^{o p} \times \mathbf{D}(\Lambda-\operatorname{Mod}-\Lambda) \rightarrow \mathbf{D}(\operatorname{Mod}-\Lambda)
$$

and

$$
-\otimes_{\Lambda}^{\mathbf{L}}-: \mathbf{D}(\operatorname{Mod}-\Lambda) \times \mathbf{D}^{-}(\Lambda-\operatorname{Mod}) \rightarrow \mathbf{D}\left(\mathbb{Z}_{p}-\operatorname{Mod}\right),
$$

respectively

$$
-\otimes_{\Lambda}^{\mathbf{L}}-: \mathbf{D}(\Lambda-\operatorname{Mod}-\Lambda) \times \mathbf{D}^{-}(\Lambda-\operatorname{Mod}) \rightarrow \mathbf{D}(\Lambda-\operatorname{Mod}) .
$$

RHom, respectively $\otimes^{\mathbf{L}}$, is computed via a projective resolution in the first, respectively second variable.

Proposition 6.1. Let $Y \in \mathbf{D}^{b}(\Lambda-M o d-\Lambda), Z \in \mathbf{D}^{b}(\Lambda-M o d)$ and let $X \in \mathbf{D}^{b}(\Lambda-M o d)$ be a bounded complex which is quasi-isomorphic to a bounded complex consisting of finitely generated free $\Lambda$-modules. Then

$$
\mathbf{R} \operatorname{Hom}_{\Lambda}\left(X, Y \otimes_{\Lambda}^{\mathbf{L}} Z\right) \cong \mathbf{R} \operatorname{Hom}_{\Lambda}(X, Y) \otimes_{\Lambda}^{\mathbf{L}} Z .
$$


Proof. (See [24, Proposition 2.1] for the case of graded algebras over a field.)

Replacing $X$ with a quasi-isomorphic complex $L \in \mathbf{D}^{b}(\Lambda$-Mod) consisting of finitely generated free $\Lambda$-modules and replacing $Z$ with a quasi-isomorphic complex $F \in \mathbf{D}^{-}(\Lambda$-Mod) consisting of projectives, we see that we have to prove

$$
\operatorname{Hom}_{\Lambda}\left(L, Y \otimes_{\Lambda} F\right)=\operatorname{Hom}_{\Lambda}(L, Y) \otimes_{\Lambda} F .
$$

But due to the boundedness condition and the fact that $L$ consists of finitely generated free modules, the $n$th module on either side becomes

$$
\bigoplus_{i, j} \operatorname{Hom}_{\Lambda}\left(L^{i}, Y^{j}\right) \otimes_{\Lambda} F^{n+i-j}
$$

while the differentials on each summand $\operatorname{Hom}_{\Lambda}\left(L^{i}, Y^{j}\right) \otimes_{\Lambda} F^{n+i-j}$ are given by

$$
\begin{gathered}
d_{L}^{i-1} \otimes 1+d_{Y}^{j} \otimes(-1)^{j-i-1}+(-1)^{n} \otimes d_{F}^{n+i-j}, \text { respectively } \\
d_{L}^{i-1} \otimes 1+d_{Y}^{j} \otimes(-1)^{n}+(-1)^{i+1} \otimes d_{F}^{n+i-j} .
\end{gathered}
$$

We will construct an isomorphism between the two complexes: If the minimal nonzero module of each of the complexes is $\operatorname{Hom}\left(L^{i_{0}}, Y^{j_{0}}\right) \otimes \Lambda F^{n_{0}+i_{0}-j_{0}}$, then the multiplication by suitable signs on the summands associated to the triple of indices $(a, b, c)=(i, j, n+i-j)$ defines an isomorphism of complexes. For example, we can choose these signs by the following rules, which determine them uniquely:

(i) $\operatorname{sign}\left(\left(i_{0}, j_{0}, n_{0}+i_{0}-j_{0}\right)\right)=1$,

(ii) $\operatorname{sign}((a+1, b, c))=\operatorname{sign}(a, b, c)$,

(iii) $\operatorname{sign}((a, b+1, c))=(-1)^{c} \operatorname{sign}((a, b, c))$,

(iv) $\operatorname{sign}((, a, b, c+1))=(-1)^{c+b+1} \operatorname{sign}((a, b, c))$.

In the proof of the next theorem we use the notation

$$
\sigma_{\geq n}(Y):=\cdots \longrightarrow 0 \longrightarrow Y^{n} / \operatorname{im}\left(Y^{n-1}\right) \longrightarrow Y^{n+1} \longrightarrow Y^{n+2} \longrightarrow \cdots
$$

for the truncation of a complex $Y$ at the degree $n$.

Theorem 6.2 (Auslander-Buchsbaum equality). For any $M \epsilon \Lambda$-mod, it holds

$$
\operatorname{pd}_{\Lambda}(M)+\operatorname{depth}_{\Lambda}(M)=\operatorname{depth}_{\Lambda}(\Lambda) .
$$

Proof. (See [24, Theorem 3.2] for the case of graded algebras over a field.) Regard $k, M, \Lambda$ as complexes concentrated in degree zero. Then the invariants in question are related to each other by the following isomorphism

$$
\mathbf{R H o m}_{\Lambda}(k, M) \cong \mathbf{R H o m}_{\Lambda}\left(k, \Lambda \otimes_{\Lambda}^{\mathbf{L}} M\right) \cong \mathbf{R H o m}_{\Lambda}(k, \Lambda) \otimes_{\Lambda}^{\mathbf{L}} M,
$$

where we use Proposition 6.1. Choosing a minimal free resolution $L$ of $M$ (see Appendix) and noting that the truncation

$$
T=\sigma_{\geq d}\left(\mathbf{R H o m}_{\Lambda}(k, \Lambda)\right)
$$

is quasi-isomorphic to $\mathbf{R H o m}_{\Lambda}(k, \Lambda)$, we can replace the right term by $T \otimes_{\Lambda} L$. 
The lowest non-zero module in $T$ is $T^{d}$ with $d=\operatorname{depth}(\Lambda)$ while the lowest non-zero module in $L$ is $L^{-\operatorname{pd}(M)}$ according to Appendix, Corollary 7.2. So the lowest non-zero module in $T \otimes_{\Lambda} L$ becomes $\left(T \otimes_{\Lambda} L\right)^{d-\operatorname{pd}(M)}=$ $T^{d} \otimes_{\Lambda} L^{-\operatorname{pd}(M)}$. Obviously, $\operatorname{depth}(M) \geq d-\operatorname{pd}(M)$, because $\operatorname{depth}(M)=$ $\min \left\{i \mid \mathrm{H}^{i}\left(\mathbf{R H o m}_{\Lambda}(k, M) \neq 0\right\}\right.$. So we need to see that $\mathrm{H}^{d-\operatorname{pd}(M)}\left(T \otimes_{\Lambda} L\right)$ is nonzero.

However, $k \cong \operatorname{Ext}_{\Lambda}^{d}(k, \Lambda)=\operatorname{ker}\left(d_{T}^{d}\right) \subseteq T^{d}$ and the "beginning" of the complex $T \otimes_{\Lambda} L$ looks like

$$
0 \longrightarrow T^{d} \otimes_{\Lambda} L^{-\operatorname{pd}(M)} \longrightarrow T^{d} \otimes_{\Lambda} L^{-\operatorname{pd}(M)+1} \oplus T^{d+1} \otimes_{\Lambda} L^{-\operatorname{pd}(M)} \longrightarrow \cdots
$$

Now it holds that

$$
0 \neq \operatorname{ker}\left(d_{T}^{d}\right) \otimes_{\Lambda} L^{-\operatorname{pd}(M)} \subseteq \operatorname{ker}\left(d_{T \otimes L}^{d-\operatorname{pd}(M)}\right)=\mathrm{H}^{d-\operatorname{pd}(M)}\left(T \otimes_{\Lambda} L\right) .
$$

Indeed, for $t \otimes l \epsilon \operatorname{ker}\left(d_{T}^{d}\right) \otimes_{\Lambda} L^{-\operatorname{pd}(M)}$, we have

$$
d_{T \otimes L}^{d-\operatorname{pd}(M)}(t \otimes l)=d_{T}^{d}(t) \otimes l+(-1)^{d-\operatorname{pd}(M)} t \otimes d_{L}^{-\operatorname{pd}(M)}(l) .
$$

The first summand is zero because $t \epsilon \operatorname{ker}\left(d_{T}^{d}\right)$ while, due to the minimality of $L$ (cf. Appendix, Proposition 7.1 (ii)), the second one lies in $\operatorname{ker}\left(d_{T}^{d}\right) \otimes_{\Lambda} \mathfrak{M} L^{d-\operatorname{pd}(M)+1} \cong$ $\Lambda / \mathfrak{M} \otimes_{\Lambda} \mathfrak{M} L^{d-\operatorname{pd}(M)+1}=0$.

Corollary 6.3. If $M$ is a finitely generated $\Lambda$-module, then

$$
\operatorname{pd}(M)=\max \left\{i \mid \mathrm{E}^{i}(M) \neq 0\right\} .
$$

Proof. Using Lemma 5.5 (ii) and local duality, we get

$$
\begin{aligned}
\operatorname{pd}(M) & =d-\operatorname{depth}(M) \\
& =d-\min \left\{i \mid \mathrm{H}_{\mathfrak{M}}^{i}(M) \neq 0\right\} \\
& =\max \left\{i \mid \mathrm{E}^{i}(M) \neq 0\right\} .
\end{aligned}
$$

Remark 6.4. The statement of the last corollary holds over an arbitrary Noetherian ring for a finitely generated modules $M$ with finite projective dimension $\operatorname{pd}_{\Lambda} M$ and can be proven directly in the following way. Consider a projective resolution of minimal length

$$
0 \longrightarrow P_{n} \stackrel{d_{n}}{\longrightarrow} P_{n-1} \stackrel{d_{n-1}}{\longrightarrow} \cdots \longrightarrow P_{0} \longrightarrow M \longrightarrow 0 .
$$

Then the $(n-1)$ th syzygy $K=\operatorname{ker}\left(d_{n-2}\right)$ has projective dimension $\operatorname{pd}_{\Lambda} K=1$, i.e. $\mathrm{D} K \simeq \mathrm{E}^{1}(K) \cong \mathrm{E}^{n}(M)$. Hence, $\mathrm{E}^{n}(M)$ cannot vanish because otherwise $K$ would be projective.

Acknowledgements. This article is based on a part of my Dissertation, Heidelberg 2000, and I would like to thank my supervisor Kay Wingberg most warmly for leading me to the nice field of "higher dimensional" Iwasawa theory. Without his advice and confidence this project would not have been possible. I am also much indebted to John Coates and Alexander Schmidt, whose comments on my manuscript helped improve the exposition. Peter Schneider is heartily acknowledged for pointing out some inaccuracies. 


\section{Appendix: Minimal resolutions}

For lack of a reference we give the proofs of some basic facts on minimal resolutions. Let $\Lambda=\mathbb{Z}_{p} \llbracket G \rrbracket$ the completed group algebra over $\mathbb{Z}_{p}$ of a finitely generated pro- $p$-group $G$ and $k=\Lambda / \mathfrak{M} \cong \mathbb{F}_{p}$ its residue class field. We assume that $\Lambda$ is Noetherian. For any finitely generated $\Lambda$-module $M$ we have the minimal representation

$$
\Lambda^{d_{0}} \stackrel{\varphi_{0}}{\longrightarrow} M
$$

with $d_{0}=\operatorname{dim}_{k} M / \mathfrak{M} M$ by the Nakayama-Lemma. Proceeding in the same manner for $\operatorname{ker}\left(\varphi_{0}\right)$ and $d_{1}=\operatorname{dim}_{k} \operatorname{ker}\left(\varphi_{0}\right) / \mathfrak{M} \operatorname{ker}\left(\varphi_{0}\right)$, we construct a minimal free resolution

$$
F_{\bullet}: \cdots \longrightarrow \Lambda^{d_{n}} \stackrel{\varphi_{n}}{\longrightarrow} \Lambda^{d_{n-1}} \stackrel{\varphi_{n-1}}{\longrightarrow} \cdots \longrightarrow \Lambda^{d_{1}} \stackrel{\varphi_{1}}{\longrightarrow} \Lambda^{d_{0}} \stackrel{\varphi_{0}}{\longrightarrow} M \longrightarrow 0 .
$$

It is easily verified that $F_{\bullet}$ is determined by $M$ up to isomorphism of complexes.

Proposition 7.1. Let $M$ be a finitely generated $\Lambda$-module and

$$
F_{\bullet}: \cdots \longrightarrow F_{n} \stackrel{\varphi_{n}}{\longrightarrow} F_{n-1} \stackrel{\varphi_{n-1}}{\longrightarrow} \cdots \longrightarrow F_{1} \stackrel{\varphi_{1}}{\longrightarrow} F_{0} \longrightarrow 0 .
$$

a free resolution of $M$. Then the following are equivalent:

(i) $F_{\bullet}$ is minimal,

(ii) $\varphi_{i}\left(F_{i}\right) \subseteq \mathfrak{M} F_{i-1}$ for all $i \geq 1$,

(iii) $\operatorname{rk}_{\Lambda}\left(F_{i}\right)=\operatorname{dim}_{k} \operatorname{Tor}_{i}^{\Lambda}(M, k)$ for all $i \geq 0$,

(iv) $\operatorname{rk}_{\Lambda}\left(F_{i}\right)=\operatorname{dim}_{k} \operatorname{Ext}_{\Lambda}^{i}(M, k)$ for all $i \geq 0$.

Proof. The equivalence of (i) and (ii) follows easily from Nakayama's lemma. Since $\operatorname{Tor}_{i}^{\Lambda}(M, k)=\mathrm{H}_{i}\left(F_{\bullet} \otimes k\right)$, (iii) holds if and only if $\varphi_{i} \otimes k=0$ for all $i \geq 0$, which is equivalent to (ii). Using $\operatorname{Ext}_{\Lambda}^{i}(M, k)=\mathrm{H}^{i}\left(\operatorname{Hom}_{\Lambda}\left(F_{\bullet}, k\right)\right)$ the equivalence of (ii) and (iv) follows similarly.

Corollary 7.2. Let $M$ be a finitely generated $\Lambda$-module. Then

$$
\begin{aligned}
\operatorname{pd}(M) & =\max \left\{i \mid F_{i} \neq 0\right\} \\
& =\max \left\{i \mid \operatorname{Tor}_{i}^{\Lambda}(M, k) \neq 0\right\} \\
& =\max \left\{i \mid \operatorname{Ext}_{\Lambda}^{i}(M, k) \neq 0\right\}
\end{aligned}
$$

\section{References}

1. M. Auslander, M. Bridger: Stable module theory, Memoirs of the AMS, vol. 94. AMS 1969

2. P.N. Balister, S. Howson: Note on Nakayama's Lemma for Compact $\Lambda$-modules, Asian J. Math. 1(2), 224-229 (1997)

3. J.-E. Björk: Rings of differential operators, North-Holland Math. Library, vol. 21. Amsterdam: North-Holland 1979 
4. J.-E. Björk: Filtered Noetherian Rings, Noetherian rings and their applications, Conf. Oberwolfach/FRG 1983. Math. Surv. Monogr. 24, 59-97 (1987)

5. J.-E. Björk: The Auslander condition on Noetherian rings, Seminaire d'algebre P. Dubreil et M.-P. Malliavin, Proc., Paris/Fr. 1987/88, LNM, vol. 1404, pp. 137173. Berlin Heidelberg New York: Springer 1989

6. A. Brumer: Pseudocompact algebras, profinite groups and class formations. J. Algebra 4, 442-470 (1966)

7. W. Bruns, J. Herzog: Cohen-Macaulay rings, Cambridge studies in advance mathematics, vol. 39. Cambridge: Cambridge University Press 1993

8. M. Chamarie: Modules sur les anneaux de Krull non commutatfs., Sém. d'Algèbre P. Dubreil et M.-P. Malliavin 1982, LNM, vol. 1029, pp. 283-310. Berlin Heidelberg New York: Springer 1983

9. J. Coates: Fragments of the $\mathrm{GL}_{2}$ Iwasawa theory of elliptic curves without complex multiplication, Arithmetic theory of elliptic curves. Lectures given at the 3rd session of the Centro Internazionale Matematico Estivo (CIME), Cetraro, Italy, July 12-19, 1997, LNM, vol. 1716, pp. 1-50. Berlin Heidelberg New York: Springer 1999

10. J. Coates, S. Howson: Euler characteristics and elliptic curves II. J. Math. Soc. Jpn. 53, 175-235 (2001)

11. J. Coates, R. Sujatha: Structure Theorem, private communication (2001)

12. J.D. Dixon, M.P.F. du Sautoy, A. Mann, D. Segal: Analytic pro-p groups, 1st ed., London Mathematical Society Lecture Note, vol. 157. Cambridge: Cambridge University Press 1991

13. R. Fossum: Duality over Gorenstein rings. Math. Scand. 26, 177-199 (1970)

14. S.I. Gelfand, Y.I. Manin: Methods of homological algebra. Berlin Heidelberg New York: Spinger 1996

15. K.R. Goodearl, R.B. Warfield: An Introduction to Noncommutative Noetherian Rings, LMS Student texts, vol. 16. Cambridge: Cambridge University Press 1989

16. M. Harris: $p$-adic representations arising from descent on Abelian varieties. Compos. Math. 39, 177-245 (1979)

17. R. Hartshorne: Residues and duality, LNM, vol. 20. Berlin Heidelberg New York: Springer 1966

18. S. Howson: Iwasawa theory of Elliptic Curves for $p$-adic Lie extensions, Ph.D. thesis, University of Cambridge, July 1998

19. S. Howson: Euler Characteristics as Invariants of Iwasawa Modules, preprint (2000)

20. S. Howson: Structure of Central Torsion Iwasawa Modules, preprint (2001)

21. U. Jannsen: Continuous Étale Cohomology. Math. Ann. 280, 207-245 (1988)

22. U. Jannsen: Iwasawa modules up to isomorphism. Adv. Stud. Pure Math. 17, 171-207 (1989)

23. U. Jannsen: A spectral sequence for Iwasawa adjoints, unpublished (1994)

24. P. Joergensen: Noncommutative graded homological identities. J. Lond. Math. Soc., II. Ser. 57, 336-350 (1998)

25. M. Lazard: Groupes analytiques p-adiques. Publ. Math. I.H.E.S. 26, 389-603 (1965)

26. T. Levasseur: Grade des modules sur certains anneaux filtres. Commun. Algebra 9(15), 1519-1532 (1981)

27. J. Neukirch, A. Schmidt, K. Wingberg: Cohomology of number fields, Grundlehren der mathematischen Wissenschaften, vol. 323. Berlin Heidelberg New York: Springer 2000

28. A. Neumann: Completed group algebras without zero divisors. Arch. Math. 51, 496-499 (1988)

29. Y. Ochi, O. Venjakob: On the structure of Selmer groups over p-adic Lie extensions. J. Algebraic Geometry (to appear)

30. J.J. Rotman: An introduction to homological algebra, Pure and Applied Mathematics, vol. 85. New York: Academic Press 1979

31. P. Schneider: Notes on modules modulo pseudo-isomorphism, private communication (2001)

32. P. Schneider, J. Teitelbaum: Banach space representations and iwasawa theory, preprint (2000) 
33. R.G. Swan: Algebraic K-theory, LNM, vol. 76. Berlin Heidelberg New York: Springer 1968

34. O. Venjakob: Iwasawa Theory of $p$-adic Lie Extensions, Dissertation, University of Heidelberg (2000)

35. O. Venjakob: Iwasawa Theory of $p$-adic Lie Extensions. preprint (2001)

36. C.A. Weibel: An introduction to homological algebra, Cambridge Studies in Advanced Mathematics, vol. 38. Cambridge: Cambridge University Press 1995

37. J.S. Wilson: Profinite groups, London Mathematical Society Monographs New Series, vol. 19. Oxford: Oxford University Press 1998

38. A. Zaks: Injective dimension of semi-primary rings. J. Algebra 13, 73-86 (1969) 